\title{
Stabilization of the 2D incompressible Euler system in an infinite strip
}

\author{
Hayk Nersisyan \\ CNRS UMR 8088, Département de Mathématiques, Université de Cergy-Pontoise, Site de Saint-Martin, 2 avenue Adolphe Chauvin, \\ F95302 Cergy-Pontoise Cedex, France
}

Received 19 September 2011; received in revised form 9 May 2012; accepted 18 December 2012

Available online 8 January 2013

\begin{abstract}
The paper is devoted to the study of a stabilization problem for the 2D incompressible Euler system in an infinite strip with boundary controls. We show that for any stationary solution $(c, 0)$ of the Euler system there is a control which is supported in a given bounded part of the boundary of the strip and stabilizes the system to $(c, 0)$.
\end{abstract}

(c) 2013 Elsevier Masson SAS. All rights reserved.

\section{Introduction}

We consider the incompressible two-dimensional Euler system

$$
\dot{u}+\langle u, \nabla\rangle u+\nabla p=0, \quad \operatorname{div} u=0,
$$

where $u=\left(u_{1}, u_{2}\right)$ and $p$ are unknown velocity field and pressure of the fluid, and

$$
\langle u, \nabla\rangle v=\sum_{i=1}^{3} u_{i}(t, x) \frac{\partial}{\partial x_{i}} v .
$$

The space variable $x=\left(x_{1}, x_{2}\right)$ belongs to the strip $D$ defined by

$$
D:=\left\{\left(x_{1}, x_{2}\right): x_{1} \in \mathbb{R}, x_{2} \in(-1,1)\right\} .
$$

Let us take two open intervals $(a, b),(a+d, b+d) \subset \mathbb{R}$ and denote

$$
\Gamma_{0}=(a, b) \times\{1\} \cup(a+d, b+d) \times\{-1\} .
$$

The aim of this paper is the study of stabilization of (1.1) with boundary controls supported by $\Gamma_{0}$. System (1.1) is completed with the boundary and initial conditions

$$
\begin{aligned}
& u \cdot n=0 \quad \text { on } \Gamma \backslash \Gamma_{0}, \\
& u(x, 0)=u_{0}(x),
\end{aligned}
$$

where $\Gamma:=\partial D$ and $n$ is the outward unit normal vector on $\Gamma$. In particular, (1.4) is equivalent to $u_{2}=0$ on $\Gamma \backslash \Gamma_{0}$.

E-mail address: Hayk.Nersisyan@u-cergy.fr. 
For any integer $s \geqslant 0$ we denote by $H^{s}(D)$ the space of vector functions $u=\left(u_{1}, u_{2}\right)$ whose components belong to the Sobolev space of order $s$ and by $\|\cdot\|_{s, D}$ the corresponding norm. If there is no confusion, we drop the index $D$. In the case $s=0$, we write $\|\cdot\|:=\|\cdot\|_{0}$. For any integer $s>0$ we define $\mathcal{H}^{s}(D)$ as the space of distributions $u$ in $D$ with $\nabla u \in H^{s-1}(D)$. We equip $\mathcal{H}^{s}(D)$ with the semi-norm

$$
\|u\|_{\mathcal{H}^{s}(D)}:=\|\nabla u\|_{s-1} .
$$

We denote by $\dot{H}^{s}(D)$ the quotient space $\mathcal{H}^{s}(D) / \mathbb{R}$. The following theorem is our main result.

Main result. Let $s \geqslant 4$ be an integer. Then for any constant $c \in \mathbb{R}$ and initial function $u_{0} \in H^{s}(D)$ that decays fast at infinity and satisfies the relations

$$
\operatorname{div} u_{0}=0, \quad u_{0} \cdot n=0 \quad \text { on } \Gamma \backslash \Gamma_{0}
$$

there exists a solution $(u, p) \in C\left(\mathbb{R}_{+}, C(\bar{D}) \cap \dot{H}^{s}(D)\right) \times C\left(\mathbb{R}_{+}, \dot{H}^{s}(D)\right)$ of $(1.1),(1.4)$ and (1.5) such that

$$
\lim _{t \rightarrow \infty}\left(\|u(\cdot, t)-(c, 0)\|_{L^{\infty}}+\|\nabla u\|_{s-1}+\|\nabla p\|_{s-1}\right)=0 .
$$

For the exact statement see Theorem 3.1. In this formulation the control is not given explicitly, but we can assume that control acts on the system as a boundary condition on $\Gamma_{0}$.

Before turning to the ideas of the proof, let us describe in a few words some previous results on the controllability of Euler and Navier-Stokes systems. Coron [7] introduced the return method to study a stabilization problem for ODE's, then using this method he proved in [8] the exact boundary controllability of 2D incompressible Euler system in a bounded domain. Glass [14] generalized this result for 3D Euler system. Chapouly [6] using return method proved the global null controllability of the Navier-Stokes system in rectangle. Recently, Glass and Rosier [15] proved the controllability of the motion of a rigid body, which is surrounded by an incompressible fluid. The asymptotic stabilization of 2D Euler equation by stationary feedback boundary controls is studied by Coron [10] and Glass [16].

Controllability of Euler and Navier-Stokes systems with distributed controls is studied in $[2,13,19,20]$; see also the book [11] for further references.

Notice that the above papers concern the problem of controllability of the fluid in a bounded domain. In this paper, we develop Coron's return method to get the controllability of velocity of 2D Euler system in an unbounded strip. This method consists in reducing the controllability of nonlinear system to the linear one. To this end, one constructs a particular solution $(\bar{u}, \bar{p})$ of Euler system and a sequence of balls $\left\{B_{i}\right\}$ covering $\bar{D}$, such that

(P) Any ball $B_{i}$ driven by the flow of $\bar{u}$ leaves $\bar{D}$ through $\Gamma_{0}$ at some time.

Then the linearized system around $\bar{u}$ is controllable. In our case, since the domain $D$ is unbounded, the number of balls $B_{i}$ is infinite, thus we cannot construct a bounded function $\bar{u}$, whose flow moves all balls outside $D$ in a finite time. However, we can find a particular solution $\bar{u}$ such that property $(P)$ holds in infinite time. This proves the stabilization of linearized system in infinite time.

To show that controllability of linearized system implies that of the nonlinear system, we need to prove that $(P)$ also holds for any $\tilde{u}$ sufficiently close to $\bar{u}$. This is obvious in the case of bounded domain. In our case, to prove this, we need some additional properties for $\bar{u}$. In particular, we need to construct a solution $\bar{u}$, which decays at infinity faster than $1 / x_{1}^{2}$. As our particular solution $\bar{u}$ is a combination of the Green functions of the Laplacian with Neumann boundary condition, we need to prove that Green functions decay at infinity. This property is a consequence of elliptic regularity and some explicit formulas for solutions of the Laplace equation in a strip.

The paper is organized as follows. In Section 2, we give preliminaries on Poisson and Euler equations in an unbounded strip. The main results of the paper are presented in Section 3. In Section 4, we construct the particular solution $\bar{u}$. In Appendix A, we prove an auxiliary result used in Section 2.

Notation. Let $J_{T}:=[0, T)$. The space of continuous functions $u: J_{T} \rightarrow X$ is denoted by $C\left(J_{T}, X\right)$. For any integer $s \geqslant 0$ or $s=\infty$, we denote

$$
C_{b}^{s}(D)=\left\{u \in C^{s}(D):\|u\|_{L^{\infty}(D)}<\infty\right\} .
$$


We set $\dot{H}^{\infty}(D):=\bigcap_{s=0}^{\infty} \dot{H}^{s}(D)$. Define

$$
\mathcal{S}(D):=\left\{u \in L^{2}(D):\left|x_{1}\right|^{\alpha} \partial^{\beta} u\left(x_{1}, x_{2}\right) \in L^{2}(D) \text { for any } \alpha \in \mathbb{R}_{+}, \beta \in \mathbb{Z}_{+}^{2}\right\} .
$$

For a vector field $u=\left(u_{1}, u_{2}\right)$ we set

$$
\operatorname{curl} u=\partial_{1} u_{2}-\partial_{2} u_{1} .
$$

The interior of a set $K$ is denoted by $\operatorname{int}(K)$. Let $B\left(x_{0}, r\right)$ be the closed ball in $\mathbb{R}^{2}$ of radius $r$ centred at $x_{0}$. We denote by $C$ a universal constant whose value may change from line to line.

\section{Preliminaries}

In this section, we present some auxiliary results on Poisson and Euler equations in an unbounded strip. The methods used in their proofs are well known and in many cases we confine ourselves to a brief description of the main ideas.

\subsection{Poisson equations in an unbounded strip}

First, let us describe the spaces $\dot{H}^{s}(D)$.

Proposition 2.1. For any integer $s \geqslant 1$ we have

(i) The space $\dot{H}^{s}(D)$ is complete.

(ii) $\mathcal{H}^{s}(D)=\left\{u \in H_{\text {loc }}^{s}(D): \nabla u \in H^{s-1}\right\}$.

(iii) If $s \geqslant 3$, then for any $u \in \mathcal{H}^{s}(D)$ there is a constant $C$ depending on $u$ such that

$$
\left|u\left(x_{1}, x_{2}\right)\right| \leqslant C\left|x_{1}\right|+C
$$

holds for all $x \in D$.

Proof. Let $\left\{u_{n}\right\} \subset \dot{H}^{s}(D)$ be a Cauchy sequence. Then there is $v \in H^{s-1}(D)$ such that $\nabla u_{n} \rightarrow v$ in $H^{s-1}(D)$ as $n \rightarrow \infty$, and for any $\varphi \in C_{0}^{\infty}(D)$ such that $\operatorname{div} \varphi=0$, we have

$$
0=\lim _{n \rightarrow \infty}\left(\nabla u_{n}, \varphi\right)_{L^{2}}=(v, \varphi)_{L^{2}} .
$$

Hence, $v=\nabla z$, where $z \in \dot{H}^{s}(D)$. This proves that $\dot{H}^{s}(D)$ is complete. Now let us prove assertion (ii). Clearly the space in the right-hand side is contained in $\dot{H}^{s}(D)$. Let us take a function $u \in \dot{H}^{s}(D)$, a compact set $K \subset D$ and let us show that $u \in H^{s}(K)$. Take two functions $\chi, \chi_{1} \in C_{0}^{\infty}(D)$ and a compact set $K_{1} \subset D$ with $\operatorname{int}\left(K_{1}\right) \supset K$ such that $\chi=1$ in $K_{1}$ and $\chi_{1}=1$ in $\tilde{K}_{1}:=\operatorname{supp} \chi$. Then there exists $r \in \mathbb{N}$ such that $\chi_{1} u \in H^{-r}(D)$. This implies that $u \in H^{-r}\left(\tilde{K}_{1}\right)$, hence

$$
\Delta(\chi u)=2 \nabla \chi \nabla u+\chi \Delta u+u \Delta \chi \in H^{\min (-r ; s-2)}\left(\tilde{K}_{1}\right) .
$$

The elliptic regularity implies $\chi u \in H^{\min (-r+2 ; s)}(D)$, thus $u \in H^{\min (-r+2 ; s)}\left(K_{1}\right)$. Repeating this argument for a compact set $K_{2} \subset K_{1}$ with $\operatorname{int}\left(K_{2}\right) \supset K$ we can show that $u \in H^{\min (-r+4 ; s)}\left(K_{2}\right)$. Iterating this, we get $u \in H^{s}(K)$. This completes the proof of assertion (ii).

It is easy to see that (ii) implies (iii). Indeed, from (ii) we get

$$
u\left(x_{1}, x_{2}\right)=\int_{0}^{x_{1}} \partial_{1} u\left(y, x_{2}\right) \mathrm{d} y+u\left(0, x_{2}\right) .
$$

The Sobolev inequality yields (iii).

Now we summarize some facts about Poisson equation. Let us take a non-negative function $\gamma \in C_{0}^{\infty}(\mathbb{R})$ such that $\operatorname{supp} \gamma=[a, b]$ and $\gamma \neq 0$ in $(a, b)$ and define 


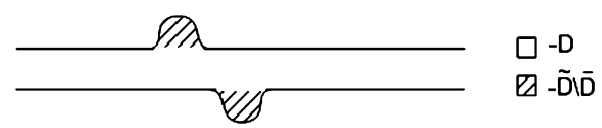

Fig. 1. Domain $\tilde{D}$.

$$
\tilde{D}:=\left\{\left(x_{1}, x_{2}\right): x_{1} \in \mathbb{R}, x_{2} \in\left(-1-\gamma\left(x_{1}-d\right), 1+\gamma\left(x_{1}\right)\right)\right\}
$$

(see Fig. 1).

Let us take $D^{\prime}=D$ or $D^{\prime}=\tilde{D}$ and consider the Dirichlet problem for the Poisson equation:

$$
\begin{aligned}
& \Delta u=f \quad \text { in } D^{\prime}, \\
& u=0 \quad \text { on } \Gamma^{\prime},
\end{aligned}
$$

where $\Gamma^{\prime}=\partial D^{\prime}$ and $f \in L^{2}\left(D^{\prime}\right)$. We say that $u \in H_{0}^{1}\left(D^{\prime}\right)$ is a solution of (2.2), (2.3) if

$$
\int_{D^{\prime}} \nabla u \nabla \theta \mathrm{d} x=-\int_{D^{\prime}} f \theta \mathrm{d} x
$$

for any $\theta \in H_{0}^{1}\left(D^{\prime}\right)$. We have the following result for the well-posedness of this problem.

Proposition 2.2. For any integer $s \geqslant 0$ and for any $f \in H^{s}\left(D^{\prime}\right)$ problem (2.2), (2.3) has a unique solution $u \in$ $H^{s+2}\left(D^{\prime}\right)$. Moreover,

$$
\|u\|_{s+2} \leqslant C\|f\|_{s},
$$

where $C$ depends only on s.

Proof. The existence of the solution $u \in H_{0}^{1}\left(D^{\prime}\right)$ is a consequence of the Riesz representation theorem. Clearly, we have

$$
\|\nabla u\|^{2} \leqslant C\|f\|\|u\| .
$$

The Poincaré inequality applied to $u\left(x_{1}, \cdot\right)$ gives

$$
\|u\| \leqslant C\left\|\partial_{2} u\right\| .
$$

Combining this with (2.5), we obtain

$$
\|u\|_{1} \leqslant C\|f\| .
$$

To show the regularity of the solution and estimate (2.4), we need the following lemma.

Lemma 2.3. For any integer $s \geqslant 1$ we have

$$
H^{s}\left(D^{\prime}\right)=\left\{z \in L^{2}\left(D^{\prime}\right): \operatorname{curl} z \in H^{s-1}\left(D^{\prime}\right), \operatorname{div} z \in H^{s-1}\left(D^{\prime}\right), z \cdot n \in H^{s-1 / 2}\left(\Gamma^{\prime}\right)\right\},
$$

where $n$ is the outward unit normal vector on $\Gamma^{\prime}$. Moreover, any function $z \in H^{s}\left(D^{\prime}\right)$ satisfies the inequality

$$
\|z\|_{s} \leqslant C\left(\|z\|+\|\operatorname{curl} z\|_{s-1}+\|\operatorname{div} z\|_{s-1}+\|z \cdot n\|_{s-1 / 2}\right),
$$

where $C$ depends only on s.

The proof of this lemma is given in Appendix A. Let us denote $z=\nabla^{\perp} u:=\left(\partial_{2} u,-\partial_{1} u\right)$. Then $\operatorname{curl} z=-\Delta u=$ $-f, \operatorname{div} z=0$. Notice that (2.3) implies that $z \cdot n=0$. It follows from Lemma 2.3 and inequality (2.6) that $z \in$ $H^{s+1}\left(D^{\prime}\right)$ and $\|z\|_{s+1} \leqslant C\|f\|_{s}$. Thus, we obtain $u \in H^{s+2}\left(D^{\prime}\right)$ and (2.4).

Let us take $g \in H^{1}\left(D^{\prime}\right)$ and consider the Neumann problem for the Poisson equation: 


$$
\begin{array}{ll}
\Delta u=\operatorname{div} g & \text { in } D^{\prime}, \\
\frac{\partial u}{\partial n}=g \cdot n & \text { on } \Gamma^{\prime} .
\end{array}
$$

We say that $u \in \dot{H}^{1}\left(D^{\prime}\right)$ is a solution of (2.7), (2.8) if for any $\theta \in H^{1}\left(D^{\prime}\right)$ we have

$$
\int_{D^{\prime}} \nabla u \nabla \theta \mathrm{d} x=\int_{D^{\prime}} g \nabla \theta \mathrm{d} x .
$$

Proposition 2.4. For any integer $s \geqslant 1$ and $g \in H^{s}\left(D^{\prime}\right)$ problem (2.7), (2.8) has a unique solution $u \in \dot{H}^{s+1}\left(D^{\prime}\right)$. Moreover,

$$
\|u\|_{\dot{H}^{s+1}} \leqslant C\|g\|_{s}
$$

Proof. The Riesz representation theorem implies the existence of the solution $u \in \dot{H}^{1}\left(D^{\prime}\right)$. Lemma 2.3 applied to $z:=\nabla u$ gives (2.9).

Now we consider the problem

$$
\begin{aligned}
& \Delta G_{a}=\partial_{1} \delta_{a} \quad \text { in } \tilde{D}, \\
& \frac{\partial G_{a}}{\partial n}=0 \quad \text { on } \partial \tilde{D},
\end{aligned}
$$

where $\delta_{a}$ is the Dirac delta function concentrated at $a=\left(a_{1}, a_{2}\right) \in \tilde{D}$.

Proposition 2.5. Problem (2.10), (2.11) has a solution $G_{a} \in C^{\infty}(\overline{\tilde{D}} \backslash\{a\})$. Moreover, the following assertions hold:

(i) For any open neighbourhood $Q$ of $a$ and for any integer $s \geqslant 1$, the solution $G_{a}$ is uniquely determined by the additional condition that it belongs to $\dot{H}^{s}(\tilde{D} \backslash \bar{Q})$.

(ii) For any $x \in \tilde{D} \backslash\{a\}$

$$
\nabla G_{a}(x)=-\frac{1}{2 \pi}\left(\frac{|x-a|^{2}-2\left(x_{1}-a_{1}\right)^{2}}{|x-a|^{4}}, \frac{-2\left(x_{1}-a_{1}\right)\left(x_{2}-a_{2}\right)}{|x-a|^{4}}\right)+\psi_{a}(x),
$$

where $\psi_{a} \in H^{\infty}(\tilde{D})$.

(iii) Let $a \in \tilde{D} \backslash \bar{D}$, then $G_{a} \in \dot{H}^{\infty}(D)$ and for any integers $1 \leqslant i, j \leqslant 2$ we have

$$
\partial_{i} \partial_{j} G_{a}\left(x_{1}, x_{2}\right) \in \mathcal{S}(D)
$$

(iv) For any fixed $x \in \tilde{D}$ the function $G_{a}(x)$ is analytic in $a \in \tilde{D} \backslash\{x\}$.

Proof. The existence of a solution $G_{a} \in C^{\infty}(\overline{\tilde{D}} \backslash\{a\})$ will be established when proving assertion (ii). To prove the uniqueness of the solution, we assume that there are two solutions $G_{1, a}$ and $G_{2, a}$. For $\tilde{G}=G_{1, a}-G_{2, a}$ we have

$$
\begin{aligned}
& \Delta \tilde{G}=0 \quad \text { in } \tilde{D}, \\
& \frac{\partial \tilde{G}}{\partial n}=0 \quad \text { on } \partial \tilde{D} .
\end{aligned}
$$

Let $\chi \in C_{0}^{\infty}(\tilde{D})$ with $\chi=1$ in $Q$. Then

$$
\Delta(\chi \tilde{G})=h,
$$

where $h \in C_{0}^{\infty}(\tilde{D})$. The elliptic regularity for a bounded domain implies that $\chi \tilde{G} \in H^{\infty}(\tilde{D})$. Since $\tilde{G} \in \dot{H}^{s}(\tilde{D} \backslash \bar{Q})$, we get $\tilde{G} \in \dot{H}^{s}(\tilde{D})$. It follows from Proposition 2.4 that $\tilde{G}=0$. 
To prove (ii), we seek the solution in the form

$$
G_{a}=\partial_{1}\left(F_{a} \chi\right)+u_{a},
$$

where $F_{a}(x)=-\frac{1}{2 \pi} \ln |x-a|$ is the fundamental solution of the Laplace operator in $\mathbb{R}^{2}, \chi \in C_{0}^{\infty}(\tilde{D}), \chi$ is 1 in a neighbourhood of $a$. Then $u_{a}$ must be the solution of the problem

$$
\begin{aligned}
& \Delta u_{a}=-\partial_{1}\left(2 \nabla F_{a} \cdot \nabla \chi+F_{a} \Delta \chi\right):=\partial_{1} f \text { in } \tilde{D}, \\
& \frac{\partial u_{a}}{\partial n}=0 \quad \text { on } \partial \tilde{D} .
\end{aligned}
$$

Since $f \in C_{0}^{\infty}(\tilde{D})$, applying Proposition 2.4 for $g=(f, 0)$, we conclude that this problem has a solution $u_{a} \in H^{\infty}(\tilde{D})$. Property (2.12) follows from the construction of $G_{a}$.

Now let us show (2.13). We have that $G_{a}$ satisfies the following problem in $D$ :

$$
\begin{array}{ll}
\Delta G_{a}=0 & \text { in } D, \\
\frac{\partial G_{a}}{\partial n}=\varphi & \text { on } \Gamma,
\end{array}
$$

where $\varphi \in C^{\infty}(\Gamma)$ and $\operatorname{supp} \varphi \subset \bar{\Gamma}_{0}$. To show that the second derivatives of the solution belong to $\mathcal{S}(D)$, let us apply the Fourier transform in $x_{1}$ to (2.15), (2.16). We obtain

$$
\begin{aligned}
& \frac{d^{2}}{d x_{2}^{2}} \hat{G}_{a}-\xi^{2} \hat{G}_{a}=0 \quad \text { in } D, \\
& \frac{d \hat{G}_{a}}{d x_{2}}(\xi,-1)=\hat{\varphi}_{1}(\xi), \\
& \frac{d \hat{G}_{a}}{d x_{2}}(\xi, 1)=\hat{\varphi}_{2}(\xi),
\end{aligned}
$$

where $\hat{G}_{a}, \hat{\varphi}_{1}$ and $\hat{\varphi}_{2}$ are Fourier transforms of $G_{a}, \varphi(\cdot,-1)$ and $\varphi(\cdot, 1)$, respectively. The solution of this ODE is given by

$$
\hat{G}_{a}\left(\xi, x_{2}\right)=\frac{\hat{\varphi}_{2}-\hat{\varphi}_{1}}{2 \xi \sinh (\xi)} \cosh \left(\xi x_{2}\right)+\frac{\hat{\varphi}_{2}+\hat{\varphi}_{1}}{2 \xi \cosh (\xi)} \sinh \left(\xi x_{2}\right) .
$$

Since $\varphi_{1}$ and $\varphi_{2}$ are compactly supported, we have

$$
\mathcal{F}\left(\partial_{i} \partial_{j} G_{a}\right) \in \mathcal{S}(D), \quad 1 \leqslant i, j \leqslant 2,
$$

whence it follows that $\partial_{i} \partial_{j} G_{a} \in S(D)$. This completes the proof of (iii).

Let $\Omega$ be any domain such that $\bar{\Omega} \subset \tilde{D}$ and $\Omega \cap(\tilde{D} \backslash \bar{D}) \neq \emptyset$. Then for any fixed $x \in \Omega$ the function $G_{a}(x)$ is analytic in $a \in \Omega \backslash\{x\}$. Indeed, let $\chi$ in (2.14) be 1 in $\Omega$. Then the analyticity of $G_{a}(x)$ is consequence of the facts that $F_{a}$ is analytic in $a$ and $u_{a}$ is a linear operator in $F_{a}$. Since $G_{a}$ is the unique solution of (2.10), (2.11), we have the analyticity of $G_{a}(x)$ in $\tilde{D} \backslash\{x\}$.

\subsection{Euler equations in an unbounded strip}

We consider the incompressible Euler system:

$$
\begin{aligned}
& \dot{u}+\langle u, \nabla\rangle u+\nabla p=0, \quad \operatorname{div} u=0 \quad \text { in } D, \\
& u \cdot n=0 \quad \text { on } \Gamma, \\
& u(x, 0)=u_{0}(x) .
\end{aligned}
$$

It is well known that if $D$ is a bounded domain or if $D=\mathbb{R}^{2}$, then problem (2.17)-(2.19) is well posed in various function spaces (e.g., see [17,18,22]).

In this subsection, we study the well-posedness of Euler system in $D$ defined by (1.2). 
Definition 2.6. For any integer $s \geqslant 3$ we say that $(u, p)$ is a solution of Euler system if $(u, p) \in C\left(J_{T}, H^{s}(D)\right) \times$ $C\left(J_{T}, \dot{H}^{s+1}(D)\right)$ and (2.17) is satisfied in the sense of distributions.

Let us show that the Euler system is equivalent to the problem

$$
\begin{aligned}
& \dot{w}+\langle u, \nabla\rangle w=0, \quad w(x, 0)=\operatorname{curl} u_{0}(x), \\
& \operatorname{curl} u=w, \quad \operatorname{div} u=0,\left.\quad u \cdot n\right|_{\Gamma}=0 .
\end{aligned}
$$

Clearly, if $(u, p)$ is a solution of the Euler system, then (2.20), (2.21) hold. Now let us show that to any solution

$$
(u, w) \in C\left(J_{T}, H^{s}(D)\right) \cap C^{1}\left(J_{T}, H^{s-1}(D)\right) \times C\left(J_{T}, H^{s-1}(D)\right)
$$

of (2.20), (2.21) there corresponds a unique solution $(u, p) \in C\left(J_{T}, H^{s}(D)\right) \times C\left(J_{T}, \dot{H}^{s+1}(D)\right)$ of (2.17)-(2.19). From (2.20) and (2.21) it follows that

$$
\operatorname{curl}(\dot{u}+\langle u, \nabla\rangle u)=0 .
$$

Hence, there exists $p \in C\left(J_{T}, \dot{H}^{s}(D)\right)$ such that $-\nabla p=\dot{u}+\langle u, \nabla\rangle u$. It is easy to see that

$$
\begin{aligned}
& -\operatorname{div} \nabla p=\operatorname{div}(\langle u, \nabla\rangle u)=\sum_{i, j=1}^{2} \partial_{i} u_{j} \partial_{j} u_{i} \in H^{s-1}, \quad \operatorname{curl} \nabla p=0, \\
& -\frac{\partial p}{\partial n}=(\langle u, \nabla\rangle u) \cdot n=\langle u, \nabla\rangle(u \cdot \tilde{n})-\sum_{i, j=1}^{2} u_{j} u_{i} \partial_{j} \tilde{n}_{i} \\
& =-\sum_{i, j=1}^{2} u_{j} u_{i} \partial_{j} \tilde{n}_{i} \in H^{s-1 / 2},
\end{aligned}
$$

where $\tilde{n}$ is a regular extension of $n$. Thus, it follows from Lemma 2.3 that $\nabla p \in C\left(J_{T}, H^{s}(D)\right)$, whence we conclude that $p \in C\left(J_{T}, \dot{H}^{s+1}(D)\right)$.

We have the following result on the local well-posedness of Euler system. The ideas used in the proof of existence of a solution play an important role in the study of stabilization problem (see Section 3). Therefore we present a rather complete proof, even though we do not really need this result.

Theorem 2.7. Let $s \geqslant 4$. For any $u_{0} \in H^{s}(D)$ satisfying the conditions

$$
\begin{aligned}
& \operatorname{div} u_{0}=0, \\
& u_{0} \cdot n=0 \quad \text { on } \Gamma,
\end{aligned}
$$

there is $T_{*}=T_{*}\left(\left\|u_{0}\right\|_{s}\right)$ such that system (2.17)-(2.19) has a unique solution $(u, p) \in C\left(J_{T_{*}}, H^{s}(D)\right) \times$ $C\left(J_{T_{*}}, \dot{H}^{s+1}(D)\right)$.

Proof. Uniqueness. To prove the uniqueness, we argue as in the case of bounded domain. We assume that there are two solutions $u_{1}$ and $u_{2}$. Then for $v=u_{1}-u_{2}$, we have

$$
\begin{aligned}
& \dot{v}+\left\langle u_{1}, \nabla\right\rangle v+\langle v, \nabla\rangle u_{2}+\nabla p=0, \\
& \operatorname{div} v=0,\left.\quad v \cdot n\right|_{\Gamma}=0, \quad v(x, 0)=0 .
\end{aligned}
$$

Multiplying (2.22) by $v$ and integrating over $D$, we get

$$
\partial_{t}\|v(\cdot, t)\|^{2} \leqslant-\int_{D}\left\langle u_{1}, \nabla\right\rangle v \cdot v \mathrm{~d} x+C\|v(\cdot, t)\|^{2}-\int_{D} \nabla p \cdot v \mathrm{~d} x,
$$

where $C>0$ is a constant depending only on $u_{2}$. Since $u_{1} \cdot n=0$, the first term on the right-hand side of (2.23) is zero. Let us show that the last term is also zero. Let us denote 


$$
\Omega_{(R)}:=\left\{x \in D:\left|x_{1}\right|<R\right\},
$$

and let $\chi \in C^{\infty}(\bar{D})$ be such that

$$
\chi(x)= \begin{cases}0, & \text { if } x \notin \bar{\Omega}_{(2)}, \\ 1, & \text { if } x \in \Omega_{(1)} .\end{cases}
$$

Clearly, we have

$$
\lim _{R \rightarrow \infty} \int_{D} \chi\left(\frac{x}{R}\right) \nabla p(x) \cdot v(x) \mathrm{d} x=\int_{D} \nabla p(x) \cdot v(x) \mathrm{d} x .
$$

On the other hand, integrating by parts, we obtain

$$
\int_{D} \chi\left(\frac{x}{R}\right) \nabla p(x) \cdot v(x) \mathrm{d} x=-\int_{\Omega_{(2 R)} \backslash \Omega_{(R)}} \nabla \chi\left(\frac{x}{R}\right) \frac{p(x)}{R} \cdot v(x) \mathrm{d} x .
$$

Since $p \in \dot{H}^{s+1}$, from assertion (iii) of Proposition 2.1 we have

$$
\sup _{x \in \Omega_{(2 R)}}\left|\frac{p(x)}{R}\right|<C,
$$

where $C$ does not depend on $R$. Thus, dominated convergence theorem yields

$$
\int_{D} \nabla p(x) \cdot v(x) \mathrm{d} x=0 .
$$

Applying the Gronwall inequality to (2.23), we obtain $v=0$.

Existence. To prove the existence of the solution, we shall need the following result.

Lemma 2.8. Let $\tilde{u} \in C\left(\mathbb{R}_{+}, H^{s}\right),\left.\tilde{u} \cdot n\right|_{\Gamma \times \mathbb{R}_{+}}=0, f \in C\left(\mathbb{R}_{+}, H^{s}\right)$ and $w_{0} \in H^{s}, s \geqslant 3$. Then the problem

$$
\begin{aligned}
& \partial_{t} w+\langle\tilde{u}, \nabla\rangle w=f, \\
& w(x, 0)=w_{0},
\end{aligned}
$$

has a unique solution $w \in C\left(\mathbb{R}_{+}, H^{s}\right)$, which satisfies the inequality

$$
\|w(\cdot, t)\|_{s} \leqslant\left\|w_{0}\right\|_{s}+\int_{0}^{t}\left(\|f(\cdot, \tau)\|_{s}+C\|w(\cdot, \tau)\|_{s}\|\nabla \tilde{u}(\cdot, \tau)\|_{s-1}\right) \mathrm{d} \tau .
$$

Proof. Let us denote by $\phi^{g}: \tilde{D} \times \mathbb{R}_{+} \rightarrow \tilde{D}$ the flow associated to $g$, i.e., the solution of the problem

$$
\begin{gathered}
\frac{\partial \phi^{g}}{\partial t}=g\left(\phi^{g}, t\right), \\
\phi^{g}(x, 0)=x .
\end{gathered}
$$

Since (2.24), (2.25) is an inhomogeneous transport equation, its solution is given by

$$
w\left(\phi^{\tilde{u}}(x, t), t\right)=w_{0}(x)+\int_{0}^{t} f\left(\phi^{\tilde{u}}(x, \tau), \tau\right) \mathrm{d} \tau .
$$

Let us derive formally inequality (2.26). Taking the $\partial^{\alpha}:=\frac{\partial^{\alpha}}{\partial x^{\alpha}},|\alpha| \leqslant s$ derivative of (2.24) and multiplying the resulting equation by $\partial^{\alpha} w$, we get 


$$
\begin{aligned}
\frac{1}{2} \frac{\mathrm{d}}{\mathrm{d} t}\left\|\partial^{\alpha} w\right\|^{2} & =\int_{D} \partial^{\alpha} f \partial^{\alpha} w \mathrm{~d} x-\int_{D} \partial^{\alpha}(\tilde{u} \cdot \nabla) w \cdot \partial^{\alpha} w \mathrm{~d} x \\
& \leqslant\left|\int_{D}(\tilde{u} \cdot \nabla) \partial^{\alpha} w \cdot \partial^{\alpha} w \mathrm{~d} x\right|+\|f\|_{s}\|w\|_{s}+C\|\nabla \tilde{u}\|_{s-1}\|w\|_{s}^{2} .
\end{aligned}
$$

Integrating by parts, one verifies that the first integral in the right-hand side vanishes. Integrating in time, we obtain (2.26).

\section{Lemma 2.9. Let $w \in H^{s}, s \geqslant 0$. Then the problem}

$$
\begin{aligned}
& \operatorname{curl} z=w, \\
& \operatorname{div} z=0, \\
& \left.z \cdot n\right|_{\Gamma}=0
\end{aligned}
$$

has a unique solution $z \in H^{s+1}$. Moreover, there is $C>0$ depending only on such that

$$
\|z\|_{s+1} \leqslant C\|w\|_{s} .
$$

Proof. Let us consider the following Dirichlet problem for the Poisson equation:

$$
\Delta v=w \text { in } D
$$$$
v=0 \quad \text { on } \Gamma \text {. }
$$

By Proposition 2.2, $v \in H^{s+2}$ and $\|v\|_{s+2} \leqslant C\|w\|_{s}$. Then for $z=-\nabla^{\perp} v$ properties (2.27)-(2.30) are satisfied.

We now return to the proof of the theorem. The proof is based on some ideas from [3] and [5].

Step 1. Let

$$
E: H^{k}(D) \rightarrow H^{k}\left(\mathbb{R}^{2}\right), \quad 0 \leqslant k \leqslant s+1
$$

be an extension operator. Let $\rho \in \mathcal{S}\left(\mathbb{R}^{2}\right)$ be the function such that

$$
\hat{\rho}(\xi)= \begin{cases}\exp \left(-\frac{|\xi|^{2}}{1-|\xi|^{2}}\right), & |\xi|<1, \\ 0, & |\xi| \geqslant 1 .\end{cases}
$$

Define $J_{m}: H^{s}(D) \rightarrow H^{s+1}(D)$ by

$$
J_{m}(v):=\left.\left(m^{2} \rho(m x) * E(v)\right)\right|_{D} .
$$

For $u_{0} \in H^{s}(D)$ we define $u_{0}^{m}:=J_{m}\left(u_{0}\right)$. Then

$$
\begin{aligned}
& u_{0}^{m} \rightarrow u_{0} \quad \text { in } H^{s}(D), \quad\left\|u_{0}^{m}\right\|_{s} \leqslant C\left\|u_{0}\right\|_{s}, \quad\left\|u_{0}^{m}\right\|_{s+1} \leqslant m C\left\|u_{0}\right\|_{s}, \\
& \left\|u_{0}^{m}-u_{0}^{k}\right\|_{s}=o(1) \quad \text { and } \quad\left\|u_{0}^{m}-u_{0}^{k}\right\|_{1}=o\left(\frac{1}{m^{s-1}}\right) \quad \text { as } m \rightarrow \infty,
\end{aligned}
$$

where (2.33) holds uniformly in $k>m$. Using Lemmas 2.8 and 2.9, we define the sequences $u^{m} \in C\left(\mathbb{R}_{+}, H^{s+1}\right)$ and $w^{m} \in C\left(\mathbb{R}_{+}, H^{s}\right)$ by

$$
\begin{aligned}
& u^{0}=u_{0}, \\
& \partial_{t} w^{m+1}+\left\langle u^{m}, \nabla\right\rangle w^{m+1}=0, \quad w^{m+1}(0)=\operatorname{curl} u_{0}^{m+1}, \\
& \operatorname{curl} u^{m+1}=w^{m+1}, \quad \operatorname{div} u^{m+1}=0,\left.\quad u^{m+1} \cdot n\right|_{\Gamma}=0 .
\end{aligned}
$$

Our strategy is to show that sequence $u^{m}$ is convergent and the limit is the solution of Euler system. From (2.26) we derive 


$$
\left\|w^{m}(\cdot, t)\right\|_{i} \leqslant\left\|\operatorname{curl} u_{0}^{m}\right\|_{i}+C_{1} \int_{0}^{t}\left\|w^{m}(\cdot, \tau)\right\|_{i}\left\|u^{m-1}(\cdot, \tau)\right\|_{i} \mathrm{~d} \tau
$$

for $i=s-1, s$.

Step 2. In this step, we show that there exists a time $T_{*}=T_{*}\left(\left\|u_{0}\right\|_{s}\right)$ such that for any $t \in J_{T_{*}}$

$$
\left\|w^{m}(\cdot, t)\right\|_{s-1} \leqslant C\left\|u_{0}^{m}\right\|_{s}, \quad\left\|w^{m}(\cdot, t)\right\|_{s} \leqslant C\left\|u_{0}^{m}\right\|_{s+1} \leqslant m C\left\|u_{0}\right\|_{s} .
$$

By induction, let us prove for $i=s-1, s$ the inequality

$$
\left\|w^{m}(\cdot, t)\right\|_{i} \leqslant y_{m}(t)
$$

where $C$ does not depend on $m$ and $y_{m}(t)$ is the solution of

$$
\dot{y}_{m}=C_{1} y_{m}^{2}, \quad y_{m}(0)=\left\|\operatorname{curl} u_{0}^{m}\right\|_{i} .
$$

Clearly (2.36) holds for $m=0$ for a sufficiently large $C$. Assume that it holds also for $m-1$ and let us prove it for $m$. From the construction of $\hat{\rho}$ we have $\left\|u_{0}^{m-1}\right\|_{i} \leqslant\left\|u_{0}^{m}\right\|_{i}$, hence $y_{m-1} \leqslant y_{m}$. Thus, from (2.34), (2.37) and induction hypothesis, we have

$$
\begin{aligned}
\left\|w^{m}(\cdot, t)\right\|_{i}-y_{m} & \leqslant C_{1} \int_{0}^{t}\left(\left\|w^{m}(\cdot, \tau)\right\|_{i}\left\|u^{m-1}(\cdot, \tau)\right\|_{i}-y_{m}^{2}\right) \mathrm{d} \tau \\
& \leqslant C_{1} \int_{0}^{t} y_{m}\left(\left\|w^{m}(\cdot, \tau)\right\|_{i}-y_{m}\right) \mathrm{d} \tau
\end{aligned}
$$

Inequality (2.36) follows from the Gronwall inequality. It is easy to see that (2.36) yields (2.35).

Step 3. Now let us show that $w^{m}$ converges in $C\left(J_{T_{*}}, H^{s-1}\right)$. In view of Lemma 2.9, sequence $u^{m}$ converges in $C\left(J_{T_{*}}, H^{s}\right)$ and the limit $u$ is the solution of Euler problem.

Notice that for $m<k$ we have

$$
\partial_{t}\left(w^{m}-w^{k}\right)+\left\langle u^{k-1}, \nabla\right\rangle\left(w^{m}-w^{k}\right)=\left\langle u^{k-1}-u^{m-1}, \nabla\right\rangle w^{m} .
$$

Denote $K^{m, k}(t):=\left\|w^{m}(\cdot, t)-w^{k}(\cdot, t)\right\|_{s-1}$. Lemma 2.8 implies

$$
\begin{aligned}
K^{m, k}(t) \leqslant & \left\|u_{0}^{m}-u_{0}^{k}\right\|_{s}+C \int_{0}^{t}\left(K^{m, k}(\tau)\left\|u^{k-1}(\cdot, \tau)\right\|_{s-1}\right. \\
& \left.+\left\|u^{m-1}(\cdot, \tau)-u^{k-1}(\cdot, \tau)\right\|_{s-1}\left\|w^{m}(\cdot, \tau)\right\|_{s}\right) \mathrm{d} \tau .
\end{aligned}
$$

On the other hand,

$$
\left\|w^{m}\right\|_{s} \leqslant C m, \quad\left\|u^{m-1}-u^{k-1}\right\|_{s-1} \leqslant\left\|u^{m-1}-u^{k-1}\right\|_{1}^{\frac{1}{s-1}}\left\|u^{m-1}-u^{k-1}\right\|_{s}^{\frac{s-2}{s-1}} .
$$

Assume for a moment that

$$
U^{m, k}:=\left\|w^{m-1}-w^{k-1}\right\| \leqslant o\left(\frac{1}{m^{s-1}}\right) .
$$

Substituting (2.40) into (2.39) and using (2.33) and (2.41), we obtain

$$
K^{m, k}(t) \leqslant o(1)+C \int_{0}^{t}\left(K^{m, k}(\tau)\left\|u^{k-1}(\cdot, \tau)\right\|_{s-1}\right) \mathrm{d} \tau .
$$

Using the Gronwall inequality, we obtain the convergence of $w^{m}$ in $C\left(J_{T_{*}}, H^{s-1}(D)\right)$. 
Step 4. To complete the proof of the theorem, it remains to show (2.41). Taking the scalar product of (2.38) with $w^{m}-w^{k}$ in $L^{2}$, we get

$$
U^{m, k}(t) \leqslant C\left\|u_{0}^{m}-u_{0}^{k}\right\|_{1}+C \int_{0}^{t} U^{m-1, k-1}\left(t_{1}\right) \mathrm{d} t_{1} .
$$

Iterating this inequality, one deduces

$$
\begin{aligned}
U^{m+p, k+p}(t) \leqslant & C\left\|u_{0}^{m+p}-u_{0}^{k+p}\right\|_{1}+C \int_{0}^{t} U^{m+p-1, k+p-1}\left(t_{1}\right) \mathrm{d} t_{1} \\
\leqslant & C\left\|u_{0}^{m+p}-u_{0}^{k+p}\right\|_{1}+C \int_{0}^{t}\left(C\left\|u_{0}^{m+p-1}-u_{0}^{k+p-1}\right\|_{1}+C \int_{0}^{t_{1}} U^{m+p-2, k+p-2}\left(t_{2}\right)\right) \mathrm{d} t_{2} \mathrm{~d} t_{1} \\
\leqslant & C\left\|u_{0}^{m+p}-u_{0}^{k+p}\right\|_{1}+C \int_{0}^{t}\left(C\left\|_{0}^{m+p-1}-u_{0}^{k+p-1}\right\|_{1}+\cdots\right. \\
& \left.+C \int_{0}^{t_{p-1}}\left(C\left\|u_{0}^{m+1}-u_{0}^{k+1}\right\|_{1}+C \int_{0}^{t_{p}} U^{m, k}\left(t_{p}\right)\right)\right) \mathrm{d} t_{p} \cdots \mathrm{d} t_{2} \mathrm{~d} t_{1} .
\end{aligned}
$$

Hence, for any $t \in J_{T_{*}}$ we obtain

$$
U^{m+p, k+p} \leqslant \sum_{j=1}^{p} \frac{C^{p-j+1} T_{*}^{p-j}}{(p-j) !}\left\|u_{0}^{m+j}-u_{0}^{k+j}\right\|_{1}+\frac{C^{p+1} T_{*}^{p}}{p !} \max _{t \in\left[0, T_{*}\right]} U^{m, k} .
$$

Since

$$
\sum_{j=1}^{\infty} \frac{C^{j+1} T_{*}^{j}}{j !}<\infty
$$

inequalities (2.33) and (2.42) imply (2.41).

Remark 2.10. We have the following assertions:

- Adapting the Beale-Kato-Majda criterion (see [4]) for an unbounded strip, one can prove that the solution of (2.17)-(2.19) is global in time. However, we shall not need this result.

- Let us take any non-zero function $g \in H^{s-1 / 2}(\Gamma)$. If the homogeneous boundary condition (2.18) is replaced by $\left.u \cdot n\right|_{\Gamma}=g$, then, the result of Theorem 2.7 holds if we add the boundary condition

$$
\operatorname{curl} u=\phi \quad \text { where } u \cdot n<0 .
$$

See [23] for the case of a bounded domain.

\section{Main result}

Let $D$ and $\Gamma_{0}$ be defined by (1.2) and (1.3). Consider the Euler system:

$$
\begin{aligned}
& \dot{u}+\langle u, \nabla\rangle u+\nabla p=0 \quad \text { in } D \times(0, \infty), \\
& \operatorname{div} u=0, \\
& u \cdot n=0 \quad \text { on } \Gamma \backslash \Gamma_{0} \times \mathbb{R}_{+}, \\
& u(x, 0)=u_{0}(x) .
\end{aligned}
$$


For any integer $s$ we denote

$$
\mathcal{X}^{s}(D)=C\left(\mathbb{R}_{+}, C_{b}(\bar{D}) \cap \dot{H}^{s}(D)\right),
$$

and $\left\langle x_{1}\right\rangle:=\left(1+x_{1}^{2}\right)^{1 / 2}$. The following theorem is our main result.

Theorem 3.1. For any constants $\alpha, \beta>0, c \in \mathbb{R}$ and integer $s \geqslant 4$, for any initial data $u_{0} \in H^{s}(D)$ such that

$$
\begin{aligned}
& \operatorname{div} u_{0}=0, \\
& u_{0} \cdot n=0 \quad \text { on } \Gamma \backslash \Gamma_{0}, \\
& \left\|\exp \left(\alpha\left\langle x_{1}\right\rangle^{2+\beta}\right) \operatorname{curl} u_{0}\left(x_{1}, x_{2}\right)\right\|_{s-1}<\infty
\end{aligned}
$$

there is a solution $(u, p) \in \mathcal{X}^{s}(D) \times C\left(\mathbb{R}_{+}, \dot{H}^{s}(D)\right)$ of (3.1)-(3.4) with

$$
\lim _{t \rightarrow \infty}\left(\|u(\cdot, t)-(c, 0)\|_{L^{\infty}(D)}+\|\nabla u(\cdot, t)\|_{s-1}+\|\nabla p(\cdot, t)\|_{s-1}\right)=0 .
$$

As explained in the Introduction, in this formulation the control is not given explicitly, but we can assume that control acts on the system as a boundary condition on $\Gamma_{0}$. So we show that there exists control $\eta \in C^{\infty}\left(\mathbb{R}_{+}, H^{s-1 / 2}\left(\Gamma_{0}\right)\right)$ such that there is a solution of our system with $\left.u \cdot n\right|_{\Gamma_{0}}=\eta$ verifying (3.8). Moreover, we show that $\eta(x, t)=\eta_{1}(x, t)+$ $\mu(t) u_{0}(x) \cdot n(x)$, where $\eta_{1} \in C^{\infty}\left(\Gamma_{0} \times \mathbb{R}_{+}\right)$does not depend on $u_{0}, \lim _{t \rightarrow \infty}\left\|\eta_{1}(\cdot, t)\right\|=0$ and $\mu \in C_{0}^{\infty}([0, \infty))$ is a non-negative function such that $\mu(0)=1$. As we mentioned in Remark 2.10, we are not able to show that this solution is unique.

Using a standard scaling argument for Euler system, we can reduce this theorem to a small neighbourhood of the origin.

Theorem 3.2. There exists $\varepsilon>0$ such that for any $u_{0} \in H^{s}(D)$ and $c \in \mathbb{R}$ verifying (3.5)-(3.7) and

$$
\left\|u_{0}\right\|_{s}<\varepsilon, \quad|c|<\varepsilon
$$

there is a solution $(u, p) \in \mathcal{X}^{s}(D) \times C\left(\mathbb{R}_{+}, \dot{H}^{s}(D)\right)$ of (3.1)-(3.4) satisfying (3.8).

Proof of Theorem 3.1. Let $\varepsilon>0$ be the constant in Theorem 3.2. Take any $u_{0} \in H^{s}(D)$ and $c \in \mathbb{R}$ verifying (3.5)(3.7). Let $M>0$ be such that

$$
\left\|\frac{u_{0}}{M}\right\|_{s}<\varepsilon, \quad\left|\frac{c}{M}\right|<\varepsilon .
$$

By Theorem 3.2, there exists a solution $\left(u_{M}, p_{M}\right)$ of (3.1)-(3.3) with initial condition $u_{M}(0)=\frac{u_{0}}{M}$, such that

$$
\lim _{t \rightarrow \infty}\left(\left\|u_{M}(\cdot, t)-\left(\frac{c}{M}, 0\right)\right\|_{L^{\infty}(D)}+\left\|\nabla u_{M}(\cdot, t)\right\|_{s-1}+\left\|\nabla p_{M}(\cdot, t)\right\|_{s-1}\right)=0 .
$$

Then $(u, p)=\left(M u_{M}(x, M t), M^{2} p_{M}(x, M t)\right)$ is a solution of our system with $u(0)=u_{0}$ and it satisfies (3.8).

Proof of Theorem 3.2. The proof of this theorem is based on generalization of the Coron return method to the case of an unbounded strip. It consists in construction of a particular solution $(\bar{u}, \bar{p})$ of (3.1)-(3.3) such that the solution of linearized system around $(\bar{u}, \bar{p})$ verifies property (3.8). Then, in the small neighbourhood of $\bar{u}$, we construct a solution $u$ of Euler system satisfying (3.8).

Step 1. In this step, we construct a particular solution $(\bar{u}, \bar{p})$ of (3.1)-(3.3) such that any point of strip $D$, driven by the flow of $\bar{u}$, leaves $\bar{D}$ at some time. Let $\hat{D} \subset \mathbb{R}^{2}$ be the strip

$$
\hat{D}:=\left\{\left(x_{1}, x_{2}\right): x_{1} \in \mathbb{R}, x_{2} \in(-2,2)\right\} .
$$

Let us admit the proposition below, which is proved in Section 4.1. 
Proposition 3.3. There are scalar functions $\theta^{i} \in C^{1}\left(\hat{D} \times \mathbb{R}_{+}\right)$with $\nabla \theta^{i} \in \mathcal{X}^{s}(\hat{D})$, open balls $B^{i}$, a sequence $\tau_{i} \subset \mathbb{R}_{+}$, constants $L, \lambda$ and an integer $N \in \mathbb{N}$ such that the following properties are true.

1. Covering. For any integer $k \geqslant 0$, we have

$$
\begin{aligned}
& {[k, k+1] \times[-1,1] \subset \bigcup_{j=1}^{N} B^{2 k N+j},} \\
& {[-k-1,-k] \times[-1,1] \subset \bigcup_{j=1}^{N} B^{(2 k+1) N+j} .}
\end{aligned}
$$

In particular, the union of balls $B^{i}$ covers $\bar{D}$ and any square $[k, k+1] \times[-1,1]$ is covered by $N$ balls.

2. Support.

$$
\operatorname{supp} \theta^{i} \subset \hat{D} \times\left(0, \tau_{i}\right)
$$

3. Vector field. The time dependent vector field $\nabla \theta^{i}$ is divergence-free in $D$ and tangent to $\Gamma \backslash \Gamma_{0}$ and $\partial \hat{D}$ :

$$
\begin{array}{ll}
\Delta \theta^{i}=0 & \text { in } D \times\left[0, \tau_{i}\right], \\
\frac{\partial \theta^{i}}{\partial n}=0 & \text { on }\left(\Gamma \backslash \Gamma_{0}\right) \cup \partial \hat{D} \times\left[0, \tau_{i}\right] .
\end{array}
$$

4. Time decay. For any $i \geqslant 1$ we have

$$
\begin{aligned}
& \left\|\nabla \theta^{i}(\cdot, t)\right\|_{\mathcal{X}^{s}(\hat{D})} \leqslant \frac{1}{i} \quad \text { for any } t \in\left[0, \tau_{i}\right], \\
& \tau_{i} \leqslant L i .
\end{aligned}
$$

5. Flow. For any $i \geqslant 1$ and $c \in \mathbb{R}$ with $|c|<\lambda$ the flow associated with $\nabla \theta^{i}+(c, 0)$ is such that

$$
\phi^{\nabla \theta^{i}+(c, 0)}\left(B^{i}, \tau_{i}\right) \subset \hat{D} \backslash \bar{D} .
$$

Moreover, there are two closed balls $\tilde{B}_{1}, \tilde{B}_{2} \subset \hat{D} \backslash \bar{D}$ such that

$$
\bigcup_{i=1}^{\infty} \phi^{\nabla \theta^{i}+(c, 0)}\left(B^{i}, \tau_{i}\right) \subset \tilde{B}_{1} \cup \tilde{B}_{2} .
$$

Let us set $t_{0}=0$,

$$
t_{i}=2 \sum_{j=1}^{i} \tau_{j}, \quad t_{i+1 / 2}=\frac{t_{i}+t_{i+1}}{2}, \quad i \geqslant 1 .
$$

We define $\bar{\theta}$ in the following way:

$$
\begin{array}{ll}
\bar{\theta}(x, t)=\theta^{i}\left(x, t-t_{i-1}\right) & \text { for } t \in\left[t_{i-1}, t_{i-1 / 2}\right], \\
\bar{\theta}(x, t)=-\theta^{i}\left(x, t_{i}-t\right) & \text { for } t \in\left[t_{i-1 / 2}, t_{i}\right] .
\end{array}
$$

Notice that from the construction of $t_{i}$ we have $t_{i}-t_{i-1 / 2}=\tau_{i}$. Thus (3.11) shows that $\bar{\theta} \in C^{1}\left(\hat{D} \times \mathbb{R}_{+}\right)$and $\nabla \bar{\theta} \in$ $\mathcal{X}^{s}(\hat{D})$. We define

$$
\begin{aligned}
& \bar{u}:=\nabla \bar{\theta}+(c, 0), \\
& \bar{p}:=-\partial_{t} \bar{\theta}-\frac{|\nabla \bar{\theta}|^{2}}{2}-c \partial_{1} \theta .
\end{aligned}
$$

Then $(\bar{u}, \bar{p})$ is a solution of (3.1)-(3.3). Indeed, by construction, $(\bar{u}, \bar{p})$ satisfies (3.1). Properties (3.12) and (3.13) imply (3.2) and (3.3), respectively. Moreover, it follows from (3.14), (3.16) that for any $i \in \mathbb{N}$, we have 


$$
\begin{aligned}
& \phi^{\bar{u}}\left(B^{i}, t_{i-1 / 2}\right) \not \subset \bar{D}, \\
& \lim _{t \rightarrow \infty}\left(\|\bar{u}(\cdot, t)-(c, 0)\|_{L^{\infty}(D)}+\|\nabla \bar{u}(\cdot, t)\|_{s-1}\right)=0 .
\end{aligned}
$$

We deduce from (3.19) and (3.20) that

$$
\phi^{\bar{u}}\left(x, t_{i}\right)=x
$$

for any $i \geqslant 1$ and $x \in \hat{D}$. We shall need the following result, which is proved in Section 4.1.

Proposition 3.4. There is a constant $v>0$ such that the functions $\theta^{i}$ in Proposition 3.3 can be chosen in a way that, for any $u \in \mathcal{X}^{s}(\hat{D})$ satisfying the inequality

$$
\int_{0}^{\infty}\|u(t)-\bar{u}(t)\|_{s, \hat{D}} \mathrm{~d} t \leqslant v,
$$

we have $\phi^{u}\left(B^{i}, t_{i-1 / 2}\right) \subset \hat{D} \backslash \bar{D}$ for any $i \geqslant 1$.

From now on, we assume that functions $\theta^{i}$ verify this proposition.

Step 2. In this step, we construct an application $F_{u_{0}}$ such that its fixed point is a solution of our stabilization problem. First, for any constant $v>0$ let us introduce the set

$$
\begin{aligned}
\mathcal{Y}_{v}\left(u_{0}\right):= & \left\{u \in \mathcal{X}^{s}(D): \operatorname{div} u=0, \int_{0}^{\infty}\|u(t)-\bar{u}(t)\|_{s, D} \mathrm{~d} t \leqslant v,\right. \\
& \left.u(x, t) \cdot n(x)=\left(u_{0}(x) \mu(t)+\bar{u}(x, t)\right) \cdot n(x) \text { on } \Gamma \times \mathbb{R}_{+}\right\},
\end{aligned}
$$

where $\mu \in C_{0}^{\infty}([0, \infty))$ is a non-negative function such that

$$
\mu(0)=1, \quad \int_{0}^{\infty} \mu(t) \mathrm{d} t<1 .
$$

Let $D_{1}:=\mathbb{R} \times\left(-\frac{3}{2}, \frac{3}{2}\right)$ and $\pi: H^{s}(D) \rightarrow H^{s}(\hat{D})$ be any linear bounded extension operator such that supp $\pi u \subset D_{1}$ for any $u \in H^{s}(D)$. Let $\kappa^{i} \in C_{0}^{\infty}(\hat{D})$ be a partition of unity subordinate to $B^{i}$, i.e.,

$$
\begin{aligned}
& \operatorname{supp} \kappa^{i} \subset B^{i}, \\
& \sum_{i=1}^{\infty} \kappa^{i}=1 \text { in } \bar{D} .
\end{aligned}
$$

Take any $u \in \mathcal{Y}_{\nu}\left(u_{0}\right)$ and let $w^{l} \in C\left(\mathbb{R}_{+}, H^{s-1}(\hat{D})\right)$ be the solution of the linear problem

$$
\begin{aligned}
& \dot{w}^{l}+\langle\tilde{u}, \nabla\rangle w^{l}=0 \quad \text { in } \hat{D} \times \mathbb{R}_{+}, \\
& w^{l}(0)=\kappa^{l} \operatorname{curl}\left(\pi u_{0}\right),
\end{aligned}
$$

where

$$
\tilde{u}=\bar{u}+\pi(u-\bar{u}) .
$$

Take $v$ such that Proposition 3.4 holds. Since supp $w^{l}(0) \subset B_{l}$, we obtain

$$
w^{l}\left(x, t_{l-1 / 2}\right)=0 \quad \text { for any } x \in \bar{D} .
$$

For any $t \in \mathbb{R}_{+}$we define the function 


$$
w(\cdot, t)=\sum_{l=i+1}^{\infty} w^{l}(\cdot, t), \quad \text { when } t \in\left[t_{i-1 / 2}, t_{i+1 / 2}\right],
$$

where $t_{-1 / 2}:=0$ and $i \geqslant 0$. Let us show that for any $t \in\left[t_{i-1 / 2}, t_{i+1 / 2}\right]$ the sum in the right-hand side of (3.27) exists and belongs to $C\left(\mathbb{R}_{+}, H^{s-1}(D)\right)$. Applying Lemma 2.8 to (3.23), (3.24), we obtain

$$
\left\|w^{l}(t)\right\|_{s-1, \hat{D}} \leqslant C\left(\left\|\kappa^{l} \operatorname{curl}\left(\pi u_{0}\right)\right\|_{s-1, \hat{D}}+\int_{0}^{t}\|\nabla \tilde{u}(\tau)\|_{s-1, \hat{D}}\left\|w^{l}(\tau)\right\|_{s-1, \hat{D}} \mathrm{~d} \tau\right) .
$$

It follows from the Gronwall inequality and relation (3.25) that

$$
\begin{aligned}
\left\|w^{l}(t)\right\|_{s-1, \hat{D}} & \leqslant C\left\|\kappa^{l} \operatorname{curl}\left(\pi u_{0}\right)\right\|_{s-1, \hat{D}} \exp \left(C \int_{0}^{t}\|\nabla \tilde{u}(\tau)\|_{s-1, \hat{D}} \mathrm{~d} \tau\right) \\
& \leqslant C\left\|\kappa^{l} \operatorname{curl}\left(\pi u_{0}\right)\right\|_{s-1, \hat{D}} \exp \left(C \int_{0}^{t}\left(\|\nabla \bar{u}(\tau)\|_{s-1, \hat{D}}+\|\bar{u}(\tau)-\tilde{u}(\tau)\|_{s, \hat{D}}\right) \mathrm{d} \tau\right) .
\end{aligned}
$$

Using the fact that $\bar{u} \in \mathcal{X}^{s}(\hat{D})$, we get

$$
\left\|w^{l}(t)\right\|_{s-1, \hat{D}} \leqslant C\left\|\kappa^{l} \operatorname{curl}\left(\pi u_{0}\right)\right\|_{s-1, \hat{D}} \exp \left(C\left(t_{i+1 / 2}+v\right)\right)
$$

for any $t \in\left[t_{i-1 / 2}, t_{i+1 / 2}\right]$. Thus

$$
\sum_{l=i}^{\infty}\left\|w^{l}(t)\right\|_{s-1, \hat{D}} \leqslant C \exp \left(C t_{i+1 / 2}\right) \sum_{l=i}^{\infty}\left\|\kappa^{l} \operatorname{curl}\left(\pi u_{0}\right)\right\|_{s-1, \hat{D}} .
$$

Using (3.7) and assertion 1 of Proposition 3.3, we derive that the right-hand side of (3.28) is finite. Hence, $w \in$ $C\left(\left[t_{i-1 / 2}, t_{i+1 / 2}\right], H^{s-1}(\hat{D})\right)$ for any $i \geqslant 0$. Moreover, assertion (3.26) yields that $w$ is continuous at $t_{i-1 / 2}$, thus $w \in C\left(\mathbb{R}_{+}, H^{s-1}(D)\right.$ ) (we emphasize that, in general, this is not true for $\left.\hat{D}\right)$. Furthermore, we have

$$
\begin{aligned}
& \dot{w}+\langle\tilde{u}, \nabla\rangle w=0 \quad \text { in } \hat{D} \times\left[t_{i-1 / 2}, t_{i+1 / 2}\right], \\
& w(0)=\sum_{l=1}^{\infty} \kappa^{l} \operatorname{curl} \pi u_{0} \quad \text { in } \hat{D} .
\end{aligned}
$$

In Step 3, we prove that for this $w$ there exists a $v \in \mathcal{Y}_{v}\left(u_{0}\right)$ such that

$$
\operatorname{curl} v=w .
$$

For any $u \in \mathcal{Y}_{v}\left(u_{0}\right)$, let $F_{u_{0}}(u):=v$. In Step 4, we show that the mapping $F_{u_{0}}: \mathcal{Y}_{v}\left(u_{0}\right) \rightarrow \mathcal{Y}_{v}\left(u_{0}\right)$ has a fixed point. We shall prove that this fixed point is a solution of our stabilization problem.

Step 3. In this step, we prove the existence of the solution $v \in \mathcal{Y}_{v}\left(u_{0}\right)$ of (3.29). By Lemma 2.9, there is a function $z \in C\left(\mathbb{R}_{+}, H^{s}(D)\right)$ such that

$$
\begin{aligned}
& \operatorname{curl} z=w, \\
& \operatorname{div} z=0, \\
& z \cdot n=0, \\
& \|z(\cdot, t)\|_{s, D} \leqslant C\|w(\cdot, t)\|_{s-1, D} .
\end{aligned}
$$

Let us take the solution of the following problem

$\Delta \varphi=0 \quad$ in $D$,

$\frac{\partial \varphi}{\partial n}=\left(u_{0} \mu\right) \cdot n \quad$ on $\Gamma$. 
From Proposition 2.4 we have $\varphi \in C\left(\mathbb{R}_{+}, \dot{H}^{s+1}(D)\right)$ and

$$
\|\varphi(\cdot, t)\|_{\dot{H}^{s+1}(D)} \leqslant C\left\|u_{0} \mu(t)\right\|_{s, D} .
$$

Denote $v=z+\nabla \varphi+\bar{u}$. Let us show that $v \in \mathcal{Y}_{\nu}\left(u_{0}\right)$ and (3.29) is verified. Clearly

$$
\begin{aligned}
& \operatorname{curl} v=\operatorname{curl} z=w, \\
& \operatorname{div} v=\operatorname{div} z+\Delta \varphi=0, \\
& v \cdot n=\left(u_{0}(x) \mu+\bar{u}\right) \cdot n \quad \text { on } \Gamma \times \mathbb{R}_{+} .
\end{aligned}
$$

Hence, to show $v \in \mathcal{Y}_{v}\left(u_{0}\right)$, it suffices to prove for sufficiently small $u_{0}$ that

$$
\int_{0}^{\infty}\|v(t)-\bar{u}(t)\|_{s, D} \mathrm{~d} t \leqslant v
$$

It follows from the construction of $v$ that

$$
\|v(\cdot, t)-\bar{u}(t)\|_{s, D} \leqslant\|\varphi(\cdot, t)\|_{\dot{H}^{s+1}(D)}+\|z(\cdot, t)\|_{s, D} .
$$

Proposition 2.4 and (3.30) imply

$$
\int_{0}^{\infty}\|v(t)-\bar{u}(t)\|_{s, D} \mathrm{~d} t \leqslant\left\|u_{0}\right\|_{s, D} \int_{0}^{\infty} \mu(t) \mathrm{d} t+C \int_{0}^{\infty}\|w(\cdot, t)\|_{s-1, D} \mathrm{~d} t .
$$

From (3.27) we have

$$
\int_{0}^{\infty}\|w(\cdot, t)\|_{s-1, D} \mathrm{~d} t=\sum_{i=0}^{\infty} \int_{t_{i}-1 / 2}^{t_{i+1 / 2}}\left\|\sum_{l=i+1}^{\infty} w^{l}(\cdot, t)\right\|_{s-1, D} \mathrm{~d} t
$$

Applying Lemma 2.8 to $\sum_{l=i+1}^{\infty} w^{l}$, we obtain

$$
\left\|\sum_{l=i+1}^{\infty} w^{l}(x, t)\right\|_{s-1, D} \leqslant C \exp \left(C \int_{0}^{t}\|\nabla \tilde{u}(\cdot, \tau)\|_{s-1, D} \mathrm{~d} \tau\right)\left\|\sum_{l=i+1}^{\infty} \kappa^{l} \operatorname{curl} u_{0}\right\|_{s-1, D} .
$$

Thus

$$
\begin{aligned}
\int_{0}^{\infty}\|w(\cdot, t)\|_{s-1, D} \mathrm{~d} t & \leqslant C \sum_{i=0}^{\infty} \int_{t_{i}-1 / 2}^{t_{i+1 / 2}}\left\|\sum_{l=i+1}^{\infty} \kappa^{l} \operatorname{curl} u_{0}\right\|_{s-1, D} \exp \left(C \int_{0}^{t}\|\nabla \tilde{u}(\cdot, \tau)\|_{s-1, D} \mathrm{~d} \tau\right) \mathrm{d} t \\
& \leqslant C_{1} \sum_{i=0}^{\infty} \int_{t_{i}-1 / 2}^{t_{i+1 / 2}} \exp \left(C t_{i+1 / 2}\right)\left\|\operatorname{curl} u_{0}\right\|_{s-1, D \backslash \bigcup_{l=1}^{i} B_{l}} \mathrm{~d} t .
\end{aligned}
$$

Combining (3.7), (3.15), (3.18) and assertion 1 of Proposition 3.3, we get

$$
\left(t_{i+1 / 2}-t_{i-1 / 2}\right) \exp \left(C t_{i+1 / 2}\right)\left\|\operatorname{curl} u_{0}\right\|_{s-1, D \backslash \bigcup_{l=1}^{i} B_{l}} \leqslant C_{2} \frac{1}{i^{2}}
$$

for any $i>0$, where $C_{2}$ does not depend on $i$. Let $K$ be a constant such that

$$
C_{1} C_{2} \sum_{i=K}^{\infty} \frac{1}{i^{2}}<\frac{v}{2}
$$

Taking $u_{0}$ sufficiently small such that 


$$
\left\|u_{0}\right\|_{s, D}+\sum_{i=1}^{K} \int_{t_{i}-1 / 2}^{t_{i+1 / 2}} \sum_{l=i+1}^{\infty}\left\|\kappa^{l} \operatorname{curl} u_{0}\right\|_{s-1, D} \exp \left(\int_{0}^{t}\|\nabla \tilde{u}(\cdot, \tau)\|_{s-1, D} \mathrm{~d} \tau\right) \mathrm{d} t \leqslant \frac{v}{2},
$$

we get (3.31).

Step 4. In this step, we show that the mapping $F_{u_{0}}: \mathcal{Y}_{v}\left(u_{0}\right) \rightarrow \mathcal{Y}_{v}\left(u_{0}\right)$ admits a fixed point, which is the solution of our stabilization problem. Let us take a sequence $u_{0}^{m}:=J_{m}\left(u_{0}\right)$, where $J_{m}$ is the operator defined by (2.31). We have that $u_{0}^{m} \in H^{s+1}(D)$ verifies (2.32), (2.33). Take $u^{0}(x, t)=\mu(t) u_{0}(x)+\bar{u}(x, t)$. For sufficiently small $u_{0}$ we have $u^{0} \in \mathcal{Y}_{v}\left(u_{0}\right)$. Let $u^{1}=F_{u_{0}^{1}}\left(u^{0}\right)$ and let $w_{1}$ be defined as in (3.27) with $u=u^{0}$ and $u_{0}(x)=u_{0}^{1}(x)$. In this way we introduce the sequences $u^{m} \in \mathcal{X}^{s}$ and $w_{m} \in C\left(\mathbb{R}_{+}, H^{s}(D)\right)$ by the relations

$$
\left\{\begin{array}{l}
u^{m+1}=F_{u_{0}^{m+1}}\left(u^{m}\right), \\
w_{m+1} \text { defined as in (3.27) with } u=u^{m} \text { and } u_{0}=u_{0}^{m+1} .
\end{array}\right.
$$

Let us show the convergence of $w_{m}$ in $C\left(\left[0, t_{1 / 2}\right], H^{s-1}(\hat{D})\right)$. This will be proved by using the same arguments as in the proof of Theorem 2.7. It is easy to see

$$
\partial_{t}\left(w_{m}-w_{k}\right)+\left\langle\tilde{u}^{k-1}, \nabla\right\rangle\left(w_{m}-w_{k}\right)=\left\langle\tilde{u}^{k-1}-\tilde{u}^{m-1}, \nabla\right\rangle w_{m}
$$

Setting $K^{m, k}(t):=\left\|w_{m}(\cdot, t)-w_{k}(\cdot, t)\right\|_{s-1, \hat{D}}$ and using Lemmas 2.8 and 2.9, we obtain

$$
\begin{aligned}
K^{m, k}(t) \leqslant & \left\|u_{0}^{m}-u_{0}^{k}\right\|_{s}+C \int_{0}^{t}\left(K^{m, k}(\tau)\left\|\nabla \tilde{u}^{k-1}(\cdot, \tau)\right\|_{s-1}\right. \\
& \left.+\left\|\tilde{u}^{m-1}(\cdot, \tau)-\tilde{u}^{k-1}(\cdot, \tau)\right\|_{s-1}\left\|w_{m}(\cdot, \tau)\right\|_{s}\right) \mathrm{d} \tau .
\end{aligned}
$$

Let us show that for any $m \in \mathbb{N}$

$$
\sup _{t \in\left[0, t_{1 / 2}\right]}\left\|w_{m}(\cdot, t)\right\|_{s-1, \hat{D}}<C\left\|u_{0}^{m}\right\|_{s, \hat{D}},
$$

where $C$ depends only on $\|\bar{u}(t)\|_{L^{1}\left(\left(0, t_{1 / 2}\right), \dot{H}^{s}(\hat{D})\right)}$ and does not depend on $m$. From the construction of $w_{m}$, we have

$$
\begin{aligned}
& \dot{w}_{m}+\left\langle\tilde{u}^{m-1}, \nabla\right\rangle w_{m}=0 \quad \text { in } \hat{D} \times \mathbb{R}_{+}, \\
& w_{m}(0)=\sum_{l=1}^{\infty} \kappa^{l} \operatorname{curl} \pi u_{0}^{m}
\end{aligned}
$$

Applying Lemma 2.8, we get

$$
\begin{aligned}
\left\|w_{m}(t)\right\|_{s-1, \hat{D}} & \leqslant C\left(\left\|u_{0}^{m}\right\|_{s, \hat{D}}+\int_{0}^{t}\left\|w_{m}\right\|_{s-1, \hat{D}}\left\|\nabla \tilde{u}^{m-1}\right\|_{s-1, \hat{D}} \mathrm{~d} t\right) \\
& \leqslant C\left(\left\|u_{0}^{m}\right\|_{s, \hat{D}}+\int_{0}^{t}\left\|w_{m}\right\|_{s-1, \hat{D}}\left(\|\nabla \bar{u}\|_{s-1, \hat{D}}+\left\|\bar{u}-\tilde{u}^{m-1}\right\|_{s, \hat{D}}\right) \mathrm{d} t\right) .
\end{aligned}
$$

Using the Gronwall inequality and the fact that $\tilde{u}_{m-1} \in \mathcal{Y}_{v}\left(u_{0}^{m}\right)$, we derive

$$
\left\|w_{m}(t)\right\|_{s-1, \hat{D}} \leqslant C\left\|u_{0}\right\|_{s} \exp \left(\int_{0}^{t_{1 / 2}}\left(\|\nabla \bar{u}\|_{s-1, \hat{D}}+\left\|\bar{u}-\tilde{u}_{m-1}\right\|_{s, \hat{D}}\right) \mathrm{d} t\right) \leqslant C_{1},
$$


where $C_{1}$ does not depend on $m$. Thus, we obtain (3.33). The construction of $u^{m}$ implies boundedness of $\sup _{t \in\left[0, t_{1 / 2}\right]}\left\|u^{m}\right\|_{s, \hat{D}}$ uniformly in $m$. In the same way we can show that

$$
\sup _{t \in\left[0, t_{1 / 2}\right]}\left\|w_{m}(\cdot, t)\right\|_{s, \hat{D}} \leqslant C\left\|u_{0}^{m}\right\|_{s+1, \hat{D}}
$$

Combining this with (2.32) and (2.33), we get

$$
\begin{aligned}
& \left\|\tilde{u}^{m-1}(\cdot, \tau)-\tilde{u}^{k-1}(\cdot, \tau)\right\|_{s-1}\left\|w_{m}(\cdot, \tau)\right\|_{s} \\
& \quad \leqslant\left\|\tilde{u}^{m-1}(\cdot, \tau)-\tilde{u}^{k-1}(\cdot, \tau)\right\|^{1 / s}\left\|\tilde{u}^{m-1}(\cdot, \tau)-\tilde{u}^{k-1}(\cdot, \tau)\right\|_{s}^{1-1 / s}\left\|w_{m}(\cdot, \tau)\right\|_{s} \leqslant a_{m, k}
\end{aligned}
$$

for any $t \in J_{t_{1 / 2}}$, where $\sup _{k \geqslant m} a_{m, k} \rightarrow 0$ as $m \rightarrow \infty$ and $a_{m, k}$ is decreasing sequence in $m$ for any fixed $k>m$ (this properties we can obtain arguing in the same way as in Theorem 2.7). Using this with (3.32) and (3.33), for any $t \in J_{t_{1 / 2}}$ we get

$$
K^{m, k}(t) \leqslant C \int_{0}^{t}\left(K^{m-1, k-1}\left(t_{1}\right)+K^{m, k}\left(t_{1}\right)\right) \mathrm{d} t_{1}+a_{m, k} .
$$

By the Gronwall inequality, for any $t \in\left[0, t_{1 / 2}\right]$ we have

$$
\begin{aligned}
K^{m+p, k+p}(t) \leqslant & C \int_{0}^{t} K^{m+p-1, k+p-1}\left(\sigma_{1}\right) e^{C t_{1}} \mathrm{~d} \sigma_{1}+C a_{m+p, k+p} \\
\leqslant & C^{2} \int_{0}^{t} \int_{0}^{\sigma_{1}} K^{m+p-2, k+p-2}\left(\sigma_{2}\right) e^{C \sigma_{1}} e^{C \sigma_{2}} \mathrm{~d} \sigma_{2} \mathrm{~d} \sigma_{1}+C e^{C t_{1 / 2}} a_{m+p-1, k+p-1}+C a_{m+p, k+p} \\
\leqslant & C^{3} \int_{0}^{t} \int_{0}^{\sigma_{1}} \int_{0}^{\sigma_{2}} K^{m+p-3, k+p-3}\left(\sigma_{2}\right) e^{C \sigma_{1}} e^{C \sigma_{2}} e^{C \sigma_{3}} \mathrm{~d} \sigma_{3} \mathrm{~d} \sigma_{2} \mathrm{~d} \sigma_{1} \\
& +C \frac{e^{2 C t_{1 / 2}}}{2} a_{m+p-2, k+p-2}+C e^{C t_{1 / 2}} a_{m+p-1, k+p-1}+C a_{m+p, k+p} \\
\leqslant & C^{p} \int_{0}^{t} \int_{0}^{\sigma_{1}} \ldots \int_{0}^{\sigma_{p-1}} K^{m, k}\left(\sigma_{p}\right) e^{C \sigma_{1}+C \sigma_{2}+\cdots+C \sigma_{p}} \mathrm{~d} \sigma_{p} \cdots \mathrm{d} \sigma_{2} \mathrm{~d} \sigma_{1}+\sum_{j=0}^{p-1} C \frac{\left(e^{\left.C t_{1 / 2}\right)^{j}}\right.}{j !} a_{m+p-j, k+p-j} .
\end{aligned}
$$

Thus, we derive

$$
K^{m+p, k+p} \leqslant \frac{C e^{p C}}{p !} \max _{t \in[0, T]} K^{m, k}+C a_{m, k} .
$$

Hence, $w_{m}$ is a convergent sequence in $C\left(\left[0, t_{1 / 2}\right], H^{s-1}(\hat{D})\right)$. In the same way we can get the convergence of $w_{m}$ in $C\left(\left[t_{i-1 / 2}, t_{i+1 / 2}\right], H^{s-1}(\hat{D})\right)$. Finally, the fact $w_{m} \in C\left(\mathbb{R}_{+}, H^{s-1}(D)\right)$ implies that $w_{m}$ converges to some $w^{*}$ in $C\left(\mathbb{R}_{+}, H^{s-1}(D)\right)$. The convergence of $w_{m}$ implies the convergence of $u^{m}$ to some $u^{*}$ in $\mathcal{X}^{s}(D)$. We have

$$
\begin{aligned}
& \operatorname{curl} u^{*}=w^{*}, \\
& \operatorname{div} u^{*}=0, \\
& u^{*}(x, t) \cdot n(x)=\left(u_{0}(x) \mu(t)+\bar{u}(x, t)\right) n(x) \quad \text { on } \Gamma \times \mathbb{R}_{+} .
\end{aligned}
$$

Let us show that

$$
w^{*}(\cdot, t)=\sum_{l=i+1}^{\infty} w^{* l}(\cdot, t) \quad \text { for } t \in\left[t_{i-1 / 2}, t_{i+1 / 2}\right],
$$


where $w^{* l}$ is the solution of

$$
\begin{aligned}
& \partial_{t} w^{* l}+\left\langle\tilde{u}^{*}, \nabla\right\rangle w^{* l}=0 \quad \text { in } \hat{D} \times \mathbb{R}_{+}, \\
& w^{* l}(0)=\kappa^{l} \operatorname{curl}\left(\pi u_{0}\right) .
\end{aligned}
$$

To this end, recall that

$$
w_{m}(\cdot, t)=\sum_{l=i+1}^{\infty} w_{m}^{l}(\cdot, t), \quad \text { when } t \in\left[t_{i-1 / 2}, t_{i+1 / 2}\right],
$$

where $w_{m}^{l}$ is the solution of

$$
\begin{aligned}
& \dot{w}_{m}^{l}+\left\langle\tilde{u}_{m-1}, \nabla\right\rangle w_{m}^{l}=0 \quad \text { in } \hat{D} \times \mathbb{R}_{+}, \\
& w_{m}^{l}(0)=\kappa^{l} \operatorname{curl}\left(\pi u_{0}^{m+1}\right) .
\end{aligned}
$$

We have that $w_{m}^{l} \rightarrow w^{* l}$ in $C\left(\mathbb{R}_{+}, H^{s-1}(\hat{D})\right)$ uniformly with respect to $l$ as $m \rightarrow \infty$ (this can be proved in the same way as in the proof of the convergence of $w_{m}$ ). Thus we have (3.38). Clearly (3.35)-(3.40) imply that $u^{*}$ is a solution of the Euler system (3.1)-(3.3).

As in (3.28), using (3.35)-(3.40) for any $t \in\left[t_{i-1 / 2}, t_{i+1 / 2}\right]$ and (3.7), we can show that

$$
\begin{aligned}
\sum_{l=i}^{\infty}\left\|w^{* l}(t)\right\|_{s-1, \hat{D}} & \leqslant C \sum_{l=i}^{\infty} \exp \left(C i^{2}\right)\left\|\kappa^{l} \operatorname{curl}\left(\pi u_{0}\right)\right\|_{s-1, \hat{D}} \\
& \leqslant C \sum_{l=i}^{\infty} \exp \left(C i^{2}\right) \exp \left(-C i^{2+\beta}\right) .
\end{aligned}
$$

Thus

$$
\lim _{t \rightarrow \infty}\left\|u^{*}(t)-\bar{u}(t)\right\|_{s, D}=0 .
$$

Combining this with (3.21), we see that the first two terms on the left-hand side of (3.8) go to zero as $t \rightarrow \infty$. Recall that

$$
\begin{aligned}
& \Delta p^{*}=-\operatorname{div}\left(\left\langle u^{*}, \nabla\right\rangle u^{*}\right), \\
& \frac{\partial p^{*}}{\partial n}=-\left(\left\langle u^{*}, \nabla\right\rangle u^{*}\right) \cdot n .
\end{aligned}
$$

Thus, Proposition 2.4 implies $\lim _{t \rightarrow \infty}\left\|\nabla p^{*}(t)\right\|_{s-1}=0$. This completes the proof of Theorem 3.1.

\section{Construction of the particular solution}

\subsection{Proof of Proposition 3.3}

We have the following simplified version of Proposition 3.3.

Lemma 4.1. For any $x_{0} \in \bar{D}$ there exist a function $\theta \in C^{\infty}\left([0,1], \dot{H}^{s+1}(\hat{D})\right)$ and a constant $\lambda>0$ such that

$$
\begin{aligned}
& \Delta \theta=0 \quad \text { in } D \times[0,1], \\
& \frac{\partial \theta}{\partial n}=0 \quad \text { on }\left(\Gamma \backslash \Gamma_{0}\right) \times[0,1], \\
& \operatorname{supp} \theta \subset \hat{D} \times(0,1), \\
& \phi^{\nabla \theta+(c, 0)}\left(x_{0}, 1\right) \notin \bar{D} \quad \text { for any }|c|<\lambda .
\end{aligned}
$$


This lemma is proved at the end of this subsection.

Proof of Proposition 3.3. It follows from Lemma 4.1 that there are functions $\tilde{\theta}^{i} \in C^{\infty}\left([0,1], \dot{H}^{s+1}(\hat{D})\right)$ and open balls $B^{i}=B\left(x_{i}, r_{i}\right) \subset \mathbb{R}^{2}, i=1, \ldots, N$ covering the rectangle $[0,1] \times[-1,1]$ such that properties (3.11)-(3.13) and (3.16) are verified for $\tau_{i}=1$. For $i=1, \ldots, N$ let us take

$$
\begin{aligned}
& \tau_{i}:=i \sup _{t \in[0,1]}\left\|\nabla \tilde{\theta}^{i}(\cdot, t)\right\|_{s, \hat{D}}, \\
& \theta^{i}(x, t):=\frac{\tilde{\theta}^{i}\left(x, \frac{t}{\tau_{i}}\right)}{\tau_{i}} .
\end{aligned}
$$

Then $B^{i}, \tau_{i}$ and $\theta^{i}$ verify (3.9)-(3.16) for $i=1, \ldots, N$. Moreover, there are closed balls $\tilde{B}_{1}, \tilde{B}_{2} \subset \hat{D} \backslash \bar{D}$ such that

$$
\bigcup_{i=1}^{N} \phi^{\nabla \theta^{i}+(c, 0)}\left(B^{i}, \tau_{i}\right) \subset \tilde{B}_{1} \cup \tilde{B}_{2} .
$$

We denote $B^{2 k N+j}:=B\left(x_{j}, r_{j}\right)+(k, 0)$ and $B^{(2 k+1) N+j}:=B\left(x_{j}, r_{j}\right)-(k+1,0), j=1, \ldots, N$. Then properties (3.9) and (3.10) are satisfied. Let $h \in C^{\infty}([0,1])$ be such that

$$
\begin{aligned}
& h(t)=0 \quad \text { for any } t \in[0,1 / 4], \\
& h(t)=1 \quad \text { for any } t \in[3 / 4,1], \\
& |h(t)| \leqslant 1 \quad \text { for any } t \in[0,1] .
\end{aligned}
$$

For any $x=\left(x_{1}, x_{2}\right) \in \hat{D}$ and $c \in \mathbb{R}$ define

$$
\tilde{\theta}^{2 k N+j}(x, t)= \begin{cases}(-k-c) x_{1} h^{\prime}(t) & \text { for } t \in[0,1] \\ \tilde{\theta}^{j}(x, t-1) & \text { for } t \in[1,2] .\end{cases}
$$

It follows from the constructions of $\tilde{\theta}^{j}, j=1, \ldots, N$ that (3.11)-(3.13) are verified for $\tau_{i}=2$. It is easy to see that for any $t \in[0,1]$ we have

$$
\phi^{\nabla \tilde{\theta}^{2 k N+j}+(c, 0)}(x, t)=(-k-c, 0) h(t)+(c, 0) t+x .
$$

Thus $\phi^{\nabla \tilde{\theta}^{2 k N+j}+(c, 0)}\left(B^{2 k N+j}, 2\right)=\phi^{\nabla \tilde{\theta}^{j}+(c, 0)}\left(B^{j}, 1\right) \not \subset \bar{D}$, which implies (3.16) and (3.17). Notice that $\nabla \tilde{\theta}^{i} \in$ $\mathcal{X}^{s}(\hat{D})$. In order to have also (3.14) and (3.15), we define $\tau_{i}$ by (4.5) and

$$
\theta^{i}(x, t):=\frac{2 \tilde{\theta}^{i}\left(x, \frac{2 t}{\tau_{i}}\right)}{\tau_{i}} .
$$

This completes the proof.

Proof of Lemma 4.1. The proof is based on the ideas of [9, Lemma A.1].

Step 1. We denote by $\mathcal{A}$ the vector space of functions $\xi \in \dot{H}^{s+1}(\hat{D})$ with the following properties

$$
\begin{aligned}
& \Delta \xi=0 \quad \text { in } D, \\
& \frac{\partial \xi}{\partial n}=0 \quad \text { on } \Gamma \backslash \Gamma_{0}, \\
& \operatorname{supp} \xi \subset \hat{D} .
\end{aligned}
$$

First, let us show that for any $x_{0} \in D \cup \Gamma_{0}$ we have

$$
\mathbb{R}^{2}=\left\{\nabla \xi\left(x_{0}\right): \xi \in \mathcal{A}\right\} .
$$

Suppose that (4.11) does not hold. Then, there is a vector $V \in \mathbb{R}^{2}, V \neq 0$ such that

$$
V \cdot \nabla \xi\left(x_{0}\right)=0
$$


for all $\xi \in \mathcal{A}$. Let $\tilde{D}$ be the domain defined in (2.1) and let $\tilde{D} \subset D_{1}$. Take any $a \in \tilde{D} \backslash \bar{D}$, and let $G_{a}$ be the solution of (2.10), (2.11). Let $B_{1}, B_{2} \subset \tilde{D} \backslash \bar{D}$ be two open neighbourhoods of $a$ such that $\bar{B}_{1} \subset B_{2}$ and let $\rho \in C^{\infty}(\tilde{D})$ be such that

$$
\rho(x)= \begin{cases}1, & \text { if } x \notin \bar{B}_{2}, \\ 0, & x \in B_{1} .\end{cases}
$$

Clearly $\pi\left(\rho G_{a}\right) \in \mathcal{A}$, thus $V \cdot \nabla \pi\left(\rho G_{a}\right)\left(x_{0}\right)=0$. Since $x_{0} \notin \bar{B}_{2}$, we have

$$
V \cdot \nabla G_{a}\left(x_{0}\right)=0
$$

for all $a \in \tilde{D} \backslash \bar{D}$. On the other hand $G_{a}$ is analytic in $a \in \tilde{D} \backslash\left\{x_{0}\right\}$ (see Proposition 2.5(iii)). Thus, we have (4.12) for all $a \in \tilde{D} \backslash\left\{x_{0}\right\}$. Using (2.12), one can find a sequence $a_{n} \rightarrow x_{0}$ such that $V \cdot \nabla G_{a_{n}}\left(x_{0}\right) \rightarrow \infty$ as $n \rightarrow \infty$, which is a contradiction to $V \neq 0$.

Step 2. Take any $x_{0} \in D \cup \Gamma_{0}, x^{1} \in \hat{D} \backslash \bar{D}$ and let $F:[0,1] \rightarrow \hat{D}$ be a continuous function such that

$$
\begin{array}{ll}
F(t)=x_{0} & \text { for any } t \in[0,1 / 4], \\
F(t)=x^{1} & \text { for any } t \in[3 / 4,1],
\end{array}
$$$$
F(t) \notin \Gamma \backslash \Gamma_{0} \quad \text { for any } t \in[0,1] \text {. }
$$

Then for any $\varepsilon>0$ we can find $\xi_{i} \in \mathcal{A}, h_{i} \in C^{\infty}([0,1]), i=1, \ldots, k$ with $\operatorname{supp} h_{i} \subset[1 / 4,3 / 4]$ such that for $\theta(x, t):=$ $\sum_{i=1}^{k} \xi_{i}(x) h_{i}(t)$ we have

$$
\left|F(t)-\phi^{\nabla \theta}\left(x_{0}, t\right)\right|<\varepsilon
$$

for any $t \in[0,1]$. It is easy to see that there is a constant $\lambda>0$ such that for any $|c|<\lambda$

$$
\left|\phi^{\nabla \theta}\left(x_{0}, t\right)-\phi^{\nabla \theta+(c, 0)}\left(x_{0}, t\right)\right|<\varepsilon .
$$

Since $\xi_{i} \in \mathcal{A}$ and $\operatorname{supp} h_{i} \subset[1 / 4,3 / 4]$, we have (4.1)-(4.3). The construction of $F$, inequalities (4.13) and (4.14) imply $\phi^{\nabla \theta+(c, 0)}\left(x_{0}, 1\right) \notin \bar{D}$ for sufficiently small $\varepsilon>0$.

Step 3. It remains to study the case $x_{0} \in \Gamma \backslash \Gamma_{0}$. Let $y_{0} \in \Gamma_{0}$ and $k \in \mathbb{R}$ be such that $x_{0}=y_{0}+(k, 0)$. Then, the function

$$
\theta(x, t)= \begin{cases}(-c-k) x_{1} h^{\prime}(t) & \text { for } t \in[0,1 / 2] \\ 2 \theta_{y_{0}}(x, 2(t-1 / 2)) & \text { for } t \in[1 / 2,1]\end{cases}
$$

satisfies (4.1)-(4.4), where $h \in C^{\infty}([0,1 / 2])$ is any function with $h(0)=0, h(1 / 2)=1$ and $\theta_{y_{0}}$ is the function constructed in Step 2 for $y_{0} \in \Gamma_{0}$.

\subsection{Proof of Proposition 3.4}

For any $m \in \mathbb{R}_{+}$, let us denote

$$
D_{-}^{m}:=(-\infty,-m] \times[-2,2] \text { and } D_{+}^{m}:=[m,+\infty) \times[-2,2] .
$$

We shall need the following lemma.

Lemma 4.2. The functions $\theta^{i}$ constructed in the proof of Proposition 3.3 are such that there exist $\varphi^{i} \in C\left(\mathbb{R}_{+}\right)$with

$$
\begin{aligned}
& \sup _{x \in \bar{D}}\left|\phi^{\nabla \theta^{i}+(c, 0)}(x, t)-x\right| \leqslant\left[\frac{i}{2 N}\right]+M \quad \text { for any } t \in\left[0, \tau_{i}\right], \\
& \left|\nabla \theta^{i}(x, t)-\nabla \theta^{i}(y, t)\right| \leqslant \frac{\varphi^{i}(t)}{(m+1)^{2}}|x-y| \quad \text { for any } x, y \in D_{+}^{m} \text { or } x, y \in D_{-}^{m},
\end{aligned}
$$

where $\int_{0}^{\tau_{i}} \varphi^{i}(t) \mathrm{d} t \leqslant M, N$ is the integer introduced in Proposition 3.3 and $M \in \mathbb{R}$ does not depend on $i$. 
Proof. It is easy to see that (4.7) and (4.9) imply

$$
\phi^{\nabla \theta^{i}+(c, 0)}(x, t)= \begin{cases}(-k-c, 0) h\left(\frac{2 t}{\tau_{i}}\right)+(c, 0) \frac{2 t}{\tau_{i}}+x & \text { for } t \in\left[0, \tau_{i} / 2\right], \\ \phi^{\nabla \tilde{\theta}^{j}+(c, 0)}\left(x, \frac{2 t}{\tau_{i}}-1\right) & \text { for } t \in\left[\tau_{i} / 2, \tau_{i}\right],\end{cases}
$$

where $k=\left[\frac{i}{2 N}\right]$ and $j=i-2 N k$. This yields (4.16) for a sufficiently large $M$. To prove (4.17), notice that in the proof of Lemma 4.1, the functions $\theta$ can be chosen such that

$$
\left\|x_{1}^{2} \partial^{\beta} \theta\right\|_{s, \hat{D}}<C
$$

where $|\beta|=2$. Indeed, since Proposition 2.5 implies that the second order derivatives of $G_{a}$ belong to $\mathcal{S}(D)$, one can replace (4.11) by

$$
\mathbb{R}^{2}=\left\{\nabla \xi\left(x_{0}\right): \xi \in \mathcal{A} \text { and }\left\|x_{1}^{2} \partial^{\beta} \xi\right\|_{s, \hat{D}}<\infty,|\beta|=2\right\} .
$$

Hence, we can find a constant $M_{1}$ such that

$$
\sup _{i=1, \ldots, N,|\beta|=2} \int_{0}^{1}\left\|x_{1}^{2} \partial^{\beta} \tilde{\theta}^{i}(t, \cdot)\right\|_{L^{\infty}(\hat{D})} \mathrm{d} t<M_{1} .
$$

Combining this with (4.7) and (4.9), we get (4.17).

Now we return to the proof of Proposition 3.4. It suffices to show that for any $\varepsilon>0$ there is $v>0$ such that the inequality

$$
\sup _{x \in B_{i}}\left|\phi^{u}(x, t)-\phi^{\bar{u}}(x, t)\right| \leqslant \varepsilon
$$

holds for any $i \geqslant 1$ and $t \in\left[0, t_{i-1 / 2}\right]$. Let us denote

$$
\begin{aligned}
& X(t)=\phi^{u}(x, t), \\
& Y(t)=\phi^{\bar{u}}(x, t),
\end{aligned}
$$

where $x \in B^{i}$. We shall prove (4.18) in the case when $i$ is even. The proof when $i$ is odd is similar. Let $k:=\left[\frac{i}{2 N}\right]$, then

$$
B^{i} \subset[k-2, k+3] \times[-2,2] .
$$

Step 1. First let us show that to establish (4.18) it suffices to prove that

$$
|X(t)-Y(t)|<1 \quad \text { for all } t \in \mathbb{R}_{+} .
$$

It is easy to see that

$$
\begin{aligned}
\partial_{t}(X(t)-Y(t)) & =u(X(t), t)-\bar{u}(Y(t), t) \\
& =(u(X(t), t)-\bar{u}(X(t), t))+(\bar{u}(X(t), t)-\bar{u}(Y(t), t))=: I_{1}(t)+I_{2}(t) .
\end{aligned}
$$

We have that

$$
\int_{0}^{\infty}\left|I_{1}(t)\right| \mathrm{d} t \leqslant v
$$

From (3.19), (3.20), (4.16) and (4.19) it follows that

$$
Y(t) \in\left[k-2-\left[\frac{j}{2 N}\right]-M, k+3+\left[\frac{j}{2 N}\right]+M\right] \times[-2,2]
$$

for any $t \in\left[0, t_{j-1 / 2}\right]$. Hence, (4.20) implies

$$
X(t) \in\left[k-3-\left[\frac{j}{2 N}\right]-M, k+4+\left[\frac{j}{2 N}\right]+M\right] \times[-2,2] .
$$


We derive from (3.19), (3.20) and (4.17) that

$$
\int_{0}^{t_{i-1 / 2}}\left|I_{2}(t)\right| \mathrm{d} t \leqslant \int_{0}^{t_{i-1 / 2}} \Psi(t)|X(t)-Y(t)| \mathrm{d} t
$$

where

$$
\Psi(t)= \begin{cases}\frac{\varphi^{j}\left(t-t_{j-1}\right)}{\left(k-2-\left[\frac{j}{2 N}\right]-M\right)^{2}}, & t \in\left[t_{j-1}, t_{j-1 / 2}\right], \\ \frac{\varphi^{j}\left(t_{j}-t\right)}{\left(k-2-\left[\frac{j}{2 N}\right]-M\right)^{2}}, & t \in\left[t_{j-1 / 2}, t_{j}\right],\end{cases}
$$

for $j<2 N(k-3-M)$ (here we use (4.17) for $\left.m=k-3-\left[\frac{j}{2 N}\right]-M\right)$ and

$$
\Psi(t)= \begin{cases}\varphi^{j}(t), & t \in\left[t_{j-1}, t_{j-1 / 2}\right] \\ \varphi^{j}\left(t_{j}-t\right), & t \in\left[t_{j-1 / 2}, t_{j}\right]\end{cases}
$$

for $j \geqslant 2 N(k-3-M)$ (in this case we use (4.17) for $m=0$ ). Thus we have

$$
\begin{aligned}
\int_{0}^{t_{i}-1 / 2} \Psi(t) \mathrm{d} t & =\int_{0}^{t_{2 N(k-3-M)-1}} \Psi(t) \mathrm{d} t+\int_{t_{2 N(k-3-M)-1}}^{t_{i-1 / 2}} \Psi(t) \mathrm{d} t \\
& \leqslant \sum_{j=1}^{2 N(k-3-M)-1} \frac{2 M}{\left(k-2-\left[\frac{j}{2 N}\right]-M\right)^{2}}+(2 N(M+4)+1) 2 M .
\end{aligned}
$$

Integrating (4.21), using (4.22)-(4.24) and the Gronwall inequality, we obtain

$$
\begin{aligned}
\left|X\left(t_{i-1 / 2}\right)-Y\left(t_{i-1 / 2}\right)\right| & \leqslant v \exp \left(\sum_{j=1}^{2 N(k-3-M)-1} \frac{2 M}{\left(k-2-\left[\frac{j}{2 N}\right]-M\right)^{2}}+(2 N(M+4)+1) 2 M\right) \\
& \leqslant v \exp \left(\sum_{j=1}^{\infty} \frac{8 M N^{2}}{j^{2}}+(2 N(M+4)+1) 2 M\right) .
\end{aligned}
$$

Choosing $v$ such that the right-hand side of (4.25) is smaller than $\varepsilon$, we prove (4.18) for all $i$.

Step 2. To complete the proof, it remains to show (4.20). To this end, let us assume that (4.20) does not hold for some $t>0$. Denote by $\tilde{t}_{0}$ the first time such that $\left|X\left(\tilde{t}_{0}\right)-Y\left(\tilde{t}_{0}\right)\right|=1$. Hence, we have (4.20) for all $t<\tilde{t}_{0}$. Step 1 implies

$$
\left|X\left(\tilde{t}_{0}\right)-Y\left(\tilde{t}_{0}\right)\right| \leqslant v \exp \left(\sum_{j=1}^{\infty} \frac{8 M N^{2}}{j^{2}}+(2 N(M+4)+1) 2 M\right) .
$$

Since the right-hand side of (4.26) does not depend on $\tilde{t}_{0}$, choosing $v$ sufficiently small, we get (4.20).

\section{Acknowledgements}

The author would like to express deep gratitude to Armen Shirikyan for drawing his attention to this problem and for many fruitful suggestions and also to Nikolay Tzvetkov for useful remarks on the Euler system.

\section{Appendix A. Proof of Lemma 2.3}

Let us consider the space

$$
H_{0}\left(D^{\prime}\right)=\left\{z \in L^{2}\left(D^{\prime}\right): \operatorname{curl} z \in L^{2}\left(D^{\prime}\right), \operatorname{div} z \in L^{2}\left(D^{\prime}\right),\left.z \cdot n\right|_{\Gamma^{\prime}}=0\right\}
$$


endowed with the norm

$$
\|z\|_{H_{0}}=\|z\|+\|\operatorname{curl} z\|+\|\operatorname{div} z\| .
$$

Here $D^{\prime}$ is a strip or is the domain $\tilde{D}$ defined in (2.1). Recall the following result (see [12, Chapter 7, Theorem 6.1]).

Theorem A.1. The following equality holds

$$
\left\{z \in H^{1}\left(D^{\prime}\right):\left.z \cdot n\right|_{\Gamma^{\prime}}=0\right\}=H_{0} .
$$

In the case of bounded domains it is shown in [21, Appendix 1, Proposition 1.4] that

$$
H^{s}(\Omega)=\left\{z \in L^{2}(\Omega): \operatorname{curl} z \in H^{s-1}(\Omega), \operatorname{div} z \in H^{s-1}(\Omega), z \cdot n \in H^{s-1 / 2}(\partial \Omega)\right\} .
$$

Let us generalize this result to the case of domain $D^{\prime}$. We shall need the following lemma.

Lemma A.2. Let $g \in H^{1 / 2}\left(\Gamma^{\prime}\right)$. Then the problem

$$
\begin{aligned}
& \Delta u-u=0 \quad \text { in } D^{\prime}, \\
& \frac{\partial u}{\partial n}=g \quad \text { on } \Gamma^{\prime}
\end{aligned}
$$

has a unique solution $u \in H^{2}\left(D^{\prime}\right)$, which satisfies

$$
\|u\|_{2} \leqslant C\|g\|_{1 / 2} .
$$

Proof. Problem (A.2), (A.3) is equivalent to

$$
\int_{D^{\prime}} \nabla u \nabla \theta \mathrm{d} x+\int_{D^{\prime}} u \theta \mathrm{d} x=\int_{\Gamma} g \theta \mathrm{d} \sigma \quad \text { for any } \theta \in H^{1}\left(D^{\prime}\right) .
$$

Since $g \in H^{-1 / 2}\left(\Gamma^{\prime}\right)$, the Riesz representation theorem implies the existence of a unique solution $u \in H^{1}\left(D^{\prime}\right)$.

Case 1. Assume $D^{\prime}=D$, and let us prove that $u \in H^{2}(D)$. It is easy to see that $v:=\partial_{1} u$ is the solution of the problem

$$
\begin{aligned}
& \Delta v-v=0 \quad \text { in } D, \\
& \frac{\partial v}{\partial n}=\partial_{1} g \quad \text { on } \Gamma .
\end{aligned}
$$

Thus $\partial_{1} u \in H^{1}(D)$ and

$$
\left\|\partial_{1} u\right\|_{1} \leqslant C\|g\|_{1 / 2} \text {. }
$$

Combining this with the fact that $\Delta u \in H^{1}(D)$, we obtain $u \in H^{2}(D)$ and (A.4).

Case 2. Now consider the case $D^{\prime}=\tilde{D}$. Let

$$
\Omega_{1}:=\left\{x \in \tilde{D}:\left|x_{1}\right|<N\right\} \text { and } \Omega_{2}:=\left\{x \in \tilde{D}:\left|x_{1}\right|<N+1\right\},
$$

where $N$ is so large that $\tilde{D} \backslash D \subset \Omega_{1}$. Let us take some function $\chi \in C^{\infty}(\overline{\tilde{D}})$ such that

$$
\chi(x)= \begin{cases}0, & \text { if } x \notin \bar{\Omega}_{2}, \\ 1, & \text { if } x \in \Omega_{1} .\end{cases}
$$

Then $w:=\chi u$ is the solution of

$$
\begin{aligned}
& \Delta w-w=2 \nabla \chi \nabla u+\Delta \chi u=: f \quad \text { in } \Omega_{2}, \\
& \frac{\partial w}{\partial n}=: \tilde{g} \quad \text { on } \partial \Omega_{2} .
\end{aligned}
$$


It is easy to see that $f \in L^{2}\left(\Omega_{2}\right)$ and $\tilde{g} \in H^{1 / 2}\left(\partial \Omega_{2}\right)$. This implies that $w \in H^{2}\left(\Omega_{2}\right)$ (e.g., see [1]). Thus $u \in H^{2}\left(\Omega_{1}\right)$. On the other hand, from the fact $\Gamma_{0} \subset \Omega_{1}$ we derive $\left.\frac{\partial u}{\partial n}\right|_{\Gamma} \in H^{1 / 2}(\Gamma)$. Hence, using the result for $D^{\prime}=D$, we see that $u \in H^{2}(D)$. This completes the proof of Lemma A.2.

Now let us prove (A.1) for $\Omega=D^{\prime}$. Clearly the space in the left-hand side is contained in the right-hand side of (A.1). By induction, let us show the other inclusion. Assume $s=1$. Let us take some function $z$ from the right-hand side of (A.1) and consider the problem:

$$
\begin{aligned}
& \Delta p-p=0 \quad \text { in } D, \\
& \frac{\partial p}{\partial n}=z \cdot n \quad \text { on } \Gamma .
\end{aligned}
$$

By Lemma A.2, we have $p \in H^{2}\left(D^{\prime}\right)$ and $\|p\|_{2} \leqslant C\|z \cdot n\|_{1 / 2}$. Let us take $w=z-\nabla p$. Clearly $w \in H_{0}$, thus Theorem A.1 implies $w \in H^{1}\left(D^{\prime}\right)$. Hence, $z \in H^{1}\left(D^{\prime}\right)$ and

$$
\|z\|_{1} \leqslant\|w\|_{1}+\|p\|_{2} \leqslant C\left(\|z\|+\|\operatorname{curl} z\|+\|\operatorname{div} z\|+\|z \cdot n\|_{1 / 2}\right) .
$$

Now assume that (A.1) holds for $s-1$ and let us prove it for $s$. Let $\tilde{n}$ be a regular extension of $n$ in $D^{\prime}$ such that $|\tilde{n}(x)|=1$. Let us show that such an extension exists. To simplify the proof, let us assume that $d=0$ in the definition of $\tilde{D}$ (see (2.1)). We define

$$
\begin{aligned}
& \tilde{n}_{1}\left(x_{1}, x_{2}\right)=-\frac{\gamma^{\prime}\left(x_{1}\right)}{\sqrt{1+\gamma^{\prime}\left(x_{1}\right)^{2}}}+h\left(x_{1}, x_{2}\right), \\
& \tilde{n}_{2}\left(x_{1}, x_{2}\right)=\frac{x_{2}}{\left(1+\gamma\left(x_{1}\right)\right) \sqrt{1+\gamma^{\prime}\left(x_{1}\right)^{2}}},
\end{aligned}
$$

where $h \in C_{b}^{\infty}(\overline{\tilde{D}}),\left.h\right|_{\partial \tilde{D}}=0$ and $h\left(x_{1}, 0\right)=1+\frac{\gamma^{\prime}\left(x_{1}\right)}{\sqrt{1+\gamma^{\prime}\left(x_{1}\right)^{2}}}$. Then we have $\left.\left(\tilde{n}_{1}, \tilde{n}_{2}\right)\right|_{\partial \tilde{D}}=n$ and $\left|\left(\tilde{n}_{1}, \tilde{n}_{2}\right)\right|>\delta$ for sufficiently small $\delta>0$. Hence, $\tilde{n}(x)=\frac{\left(\tilde{n}_{1}, \tilde{n}_{2}\right)}{\left|\left(\tilde{n}_{1}, \tilde{n}_{2}\right)\right|}$ is an extension of $n$. Let us take $v:=\nabla^{\perp}(z \cdot \tilde{n})$. Then $v \in L^{2}$, $\operatorname{div} v=0$. Since $v \cdot \tilde{n}$ is the tangential derivative of $z \cdot \tilde{n}$ along $\Gamma^{\prime}$, we have $v \cdot \tilde{n} \in H^{s-3 / 2}\left(\Gamma^{\prime}\right)$. On the other hand

$$
-\operatorname{curl} v=\Delta(z \cdot \tilde{n})=\left(\Delta z_{1}\right) \tilde{n}_{1}+\left(\Delta z_{2}\right) \tilde{n}_{2}+\tilde{v}
$$

where $\tilde{v} \in H^{s-2}$. It follows from the facts $\Delta z_{1}=\partial_{1} \operatorname{div} z+\partial_{2} \operatorname{curl} z$ and $\Delta z_{2}=\partial_{2} \operatorname{div} z-\partial_{1} \operatorname{curl} z$ that $\operatorname{curl} v \in H^{s-2}$. Thus the induction hypothesis yields $\nabla^{\perp}(z \cdot \tilde{n}) \in H^{s-1}$. Hence,

$$
\begin{aligned}
& \left(\partial_{2} z_{1}\right) \tilde{n}_{1}+\left(\partial_{2} z_{2}\right) \tilde{n}_{2} \in H^{s-1}, \\
& \left(\partial_{1} z_{1}\right) \tilde{n}_{1}+\left(\partial_{1} z_{2}\right) \tilde{n}_{2} \in H^{s-1} .
\end{aligned}
$$

Combining this with $\operatorname{div} z \in H^{s-1}$ and $\operatorname{curl} z \in H^{s-1}$, we obtain $\tilde{n} \cdot \nabla^{\perp} z_{i} \in H^{s-1}$ and $\tilde{n} \cdot \nabla z_{i} \in H^{s-1}$ for $i=1,2$. Thus $\nabla z_{i} \in H^{s-1}$, which completes the proof.

\section{References}

[1] S. Agmon, A. Douglis, L. Nirenberg, Estimates near the boundary for solutions of elliptic partial differential equations satisfying general boundary conditions. II, Comm. Pure Appl. Math. 17 (1964) 35-92.

[2] A. Agrachev, A. Sarychev, Navier-Stokes equations controllability by means of low modes forcing, J. Math. Fluid Mech. 7 (2005) 108-152.

[3] C. Bardos, U. Frisch, Finite-time regularity for bounded and unbounded ideal incompressible fluids using Hölder estimates, Lecture Notes in Math. 565 (1976) 1-13.

[4] J.T. Beale, T. Kato, A. Majda, Remarks on the breakdown of smooth solutions for the 3-D Euler equations, Comm. Math. Phys. 94 (3) (1984) 61-66.

[5] J.L. Bona, R. Smith, The initial-value problem for the Korteweg-de Vries equation, Philos. Trans. Roy. Soc. London Ser. A 278 (1287) (1975) 555-601.

[6] M. Chapouly, On the global null controllability of a Navier-Stokes system with Navier slip boundary conditions, J. Differential Equations 247 (7) (2009) 2094-2123.

[7] J.-M. Coron, Global asymptotic stabilization for controllable systems without drift, Math. Control Signals Syst. 5 (1992) $295-312$.

[8] J.-M. Coron, On the controllability of 2-D incompressible perfect fluids, J. Math. Pures Appl. 75 (2) (1996) 155-188. 
[9] J.-M. Coron, On the controllability of the 2-D incompressible Navier-Stokes equations with the Navier slip boundary conditions, ESAIM Control Optim. Calc. Var. 1 (1996) 35-75.

[10] J.-M. Coron, On the null asymptotic stabilization of 2-D incompressible Euler equation in a simply connected domain, SIAM J. Control Optim. 37 (6) (1999) 1874-1896.

[11] J.-M. Coron, Control and Nonlinearity, Mathematical Surveys and Monographs, vol. 136, 2007.

[12] G. Duvaut, J.-L. Lions, Les inéquations en mécanique et en physique, Dunod, Paris, 1972.

[13] A.V. Fursikov, O.Yu. Imanuvilov, Exact controllability of the Navier-Stokes and Boussinesq equations, Russian Math. Surv. 54 (3) (1999) 93-146.

[14] O. Glass, Exact boundary controllability of 3-D Euler equation, ESAIM Control Optim. Calc. Var. 5 (2000) 1-44.

[15] O. Glass, L. Rosier, On the control of the motion of a boat, preprint, 2011.

[16] O. Glass, Asymptotic stabilizability by stationary feedback of the two-dimensional Euler equation: the multiconnected case, SIAM J. Control Optim. 44 (3) (2005) 1105-1147.

[17] T. Kato, On classical solutions of the two-dimensional non-stationary Euler equation, Arch. Rational Mech. Anal. 25 (1967) 188-200.

[18] T. Kato, G. Ponce, Commutator estimates and the Euler and Navier-Stokes equations, Comm. Pure Appl. Math. 41 (7) (1988) 891-907.

[19] H. Nersisyan, Controllability of 3D incompressible Euler equations by a finite-dimensional external force, ESAIM Control Optim. Calc. Var. 16 (3) (2010) 677-694.

[20] A. Shirikyan, Approximate controllability of three-dimensional Navier-Stokes equations, Comm. Math. Phys. 266 (1) (2006) $123-151$.

[21] R. Temam, Navier-Stokes Equations. Theory and Numerical Analysis, vol. 2, North-Holland Publishing Co., 1977.

[22] W. Wolibner, Un théorème sur l'existence du mouvement plan d'un fluide parfait, homogène, incompressible, pendant un temps infiniment long, Math. Z. 37 (1) (1933) 698-726.

[23] V. Yudovich, The flow of a perfect, incompressible liquid through a given region, Dokl. Akad. Nauk SSSR 146 (1962) $561-564$. 